Pacific Journal of Mathematics

THE MOD 2 EQUIVARIANT COHOMOLOGY ALGEBRAS OF
CONFIGURATION SPACES 


\title{
THE MOD 2 EQUIVARIANT COHOMOLOGY ALGEBRAS OF CONFIGURATION SPACES
}

\author{
NGUYÊN H. V. HuNG \\ Dedicated to my mother
}

\begin{abstract}
The mod 2 equivariant cohomology algebras of configuration spaces are determined by means of the Dickson characteristic classes, which are derived from the modular invariants of the general linear groups $\mathrm{GL}\left(n, \mathbb{Z}_{2}\right)$ and closely related to the Stiefel-Whitney classes.
\end{abstract}

Introduction. Let us consider the configuration space $F\left(\mathbb{R}^{q}, m\right)=$ $\left\{\left(x_{1}, \ldots, x_{m}\right): x_{i} \in \mathbb{R}^{q}, x_{i} \neq x_{j}\right.$ if $\left.i \neq j, 1 \leq i, j \leq m\right\}$ as a free $\mathfrak{S}_{m}$-space, where the action of the symmetric group $\mathfrak{S}_{m}$ of degree $m$ is given by permutations of the factors. As is well known, the limit of the spaces of orbits

$$
F\left(\mathbb{R}^{\infty}, m\right) / \mathfrak{S}_{m}=\underset{q}{\lim } F\left(\mathbb{R}^{q}, m\right) / \mathfrak{S}_{m}
$$

becomes a classifying space of $\mathfrak{S}_{m}$. Meanwhile the limit

$$
H^{*}\left(F\left(\mathbb{R}^{q}, \infty\right) / \mathfrak{S}_{\infty} ; \mathbb{Z}_{p}\right)=\underset{m}{\lim } H^{*}\left(F\left(\mathbb{R}^{q}, m\right) / \mathfrak{S}_{m} ; \mathbb{Z}_{p}\right)
$$

is equipped with the Hopf algebra structure introduced essentially by M. Nakaoka [12; §2] for $1 \leq q \leq \infty$. Here $\mathbb{Z}_{p}$ denotes the prime field of $p$ elements.

Let

$$
\begin{array}{r}
J_{\text {odd }}(q)=\left\{\left(h_{0}, \ldots, h_{n-1}\right) \neq 0 ; n>0, h_{i} \in \mathbb{Z}_{+}, h_{0}+\cdots+h_{n-1}<q,\right. \\
\text { there exists } \left.j \text { such that } h_{j} \text { is odd }\right\}
\end{array}
$$

for $1 \leq q \leq \infty$. Then, in [13], [15] for each $H=\left(h_{0}, \ldots, h_{n-1}\right) \in$ $J_{\text {odd }}(\infty)$ we have introduced the universal Dickson characteristic class

$$
W^{H} \in H^{*}\left(\mathfrak{S}_{\infty} ; \mathbb{Z}_{2}\right)=\underset{m}{\lim } H^{*}\left(\mathfrak{S}_{m} ; \mathbb{Z}_{2}\right)
$$

of degree $\operatorname{dim}\left(W^{H}\right)=\sum_{s=0}^{n-1} h_{s}\left(2^{n}-2^{s}\right)$. Here we use the name of L. E. Dickson because these classes are related to his modular invariant theory as seen in [15]. We have proved the following. 
TheOREM A [13; 3.4], [15; 4.10]. As algebras we have

$$
H^{*}\left(\mathfrak{S}_{\infty} ; \mathbb{Z}_{2}\right)=\mathbb{Z}_{2}\left[W^{H} ; H \in J_{\text {odd }}(\infty)\right]
$$

A direct consequence of this theorem is the determination of the algebra $H^{*}\left(\mathfrak{S}_{m} ; \mathbb{Z}_{2}\right)$ for every $m$ by means of the well-known Steenrod epimorphism $H^{*}\left(\mathfrak{S}_{\infty} ; \mathbb{Z}_{2}\right) \rightarrow H^{*}\left(\mathfrak{S}_{m} ; \mathbb{Z}_{2}\right)$. Nakaoka [12], of course, computed $H^{*}\left(\mathfrak{S}_{\infty} ; \mathbb{Z}_{2}\right)$ as a Hopf algebra. Our result is a new description of the generators in the framework of the invariant theory.

The spaces $F\left(\mathbb{R}^{q}, m\right) / \mathfrak{S}_{m}$ have been studied by many authors (cf. E. Fadell-L. Neuwirth [4], J. P. May [9], G. Segal [19], D. B. Fuks [5], F. Cohen [2], Huỳnh Mùi [7]) because of their deep relations to the symmetric groups, the iterated loop spaces and the homotopy of the spheres. In particular, F. R. Cohen ([2], p. 226-231, 237-243) gave a map $\theta: F\left(R^{q}, \infty\right) / \mathfrak{S}_{\infty} \rightarrow \Omega_{0}^{q} S^{q}$ which induces an isomorphism in homology as Hopf algebras. Moreover, Huỳnh Mùi in [7; 10.8] has computed the modules $H^{*}\left(F\left(\mathbb{R}^{q}, m\right) / \mathfrak{S}_{m} ; \mathbb{Z}_{p}\right)$ by use of H. Cartan's works on $H^{*}\left(K(\mathbb{Z}, q) ; \mathbb{Z}_{p}\right)$ and the classical Steenrod decomposition theorem.

In the present paper, we shall determine the algebras

$$
H^{*}\left(F\left(\mathbb{R}^{q}, m\right) / \mathfrak{S}_{m} ; \mathbb{Z}_{p}\right)
$$

for $p=2$ by means of Theorem A. For $p>2$, these algebras will be studied in a subsequent paper by means of our result on the algebra $H^{*}\left(\mathfrak{S}_{\infty} ; \mathbb{Z}_{p}\right)$ (see $[16, \mathbf{1 6 b}$ for our result with $p>2$, and $\left.16 \mathrm{c}]\right)$. So from now on, the coefficient ring is always assumed to be $\mathbb{Z}_{2}$.

According to Huỳnh Mùi $[7 ; 10.8]$, the canonical inclusion $i(F, q)$ : $F\left(\mathbb{R}^{q}, \infty\right) / \mathfrak{S}_{\infty} \rightarrow F\left(\mathbb{R}^{\infty}, \infty\right) / \mathfrak{S}_{\infty}$ induces the epimorphism

$$
i^{*}(F, q): H^{*}\left(\mathfrak{S}_{\infty}\right)=H^{*}\left(F\left(\mathbb{R}^{\infty}, \infty\right) / \mathfrak{S}_{\infty}\right) \rightarrow H^{*}\left(F\left(\mathbb{R}^{q}, \infty\right) / \mathfrak{S}_{\infty}\right) .
$$

As we shall see later, for $H \in J_{\text {odd }}(q)$

$$
i^{*}(F, q) W^{H} \neq 0 \Leftrightarrow H \in J_{\text {odd }}(q) .
$$

Let us simply denote $i^{*}(F, q) W^{H}$ by $W^{H}$. Now we can state the main result of this paper as follows.

Theorem C. As algebras we get for $1 \leq q \leq \infty$

$$
\begin{aligned}
& H^{*}\left(F\left(\mathbb{R}^{q}, \infty\right) / \mathfrak{S}_{\infty}\right) \\
& \quad=\mathbb{Z}_{2}\left[W^{H} ; H \in J_{\text {odd }}(q)\right] /\left(\left(W^{H}\right)^{2^{h(q, H)}} ; H \in J_{\text {odd }}(q)\right) .
\end{aligned}
$$


Here

$$
\begin{aligned}
& h(q, H)=\min \left\{h \in \mathbb{N} ; 2^{h}\left(h_{0}+\cdots+h_{n-1}\right) \geq q\right\} \\
& \qquad \text { for } H=\left(h_{0}, \ldots, h_{n-1}\right) .
\end{aligned}
$$

For $q=2$, this result was obtained by D. B. Fuks [5] and independently by G. Segal (see E. Brieskorn [1]). A result of this sort can also quickly be derived from [21], as the referee notes at the end of this introduction. However, it should be noted that the brief form [14] of this paper appeared exactly at the same time as [21]. Furthermore, our result which was derived from the invariant theory is much useful, for instance, in computing the action of the Steenrod algebra on the cohomology of configuration spaces, because it allows us to avoid the Nishida relations. (It is shown in [16c] and our subsequent paper.)

Again, by the Steenrod decomposition theorem we have the epimorphism $H^{*}\left(F\left(\mathbb{R}^{q}, \infty\right) / \mathfrak{S}_{\infty}\right) \rightarrow H^{*}\left(F\left(\mathbb{R}^{q}, m\right) / \mathfrak{S}_{m}\right) \quad$ (see Huỳnh Mùi [7; 3.2 and 10.8]). From this we determine the algebras $H^{*}\left(F\left(\mathbb{R}^{q}, m\right) / \mathfrak{S}_{m}\right)$ as an application of Theorem $\mathrm{C}$.

We shall define the wreath product $M(q, n)$ of projective spaces and imbed it in the space $F\left(\mathbb{R}^{q}, 2^{n}\right) / \mathfrak{S}_{2^{n}}$. As a consequence $M(\infty, n)$ $=\lim _{q} M(q, n)$ is a classifying space of the Sylow 2-subgroup $\mathfrak{S}_{2^{n}, 2}$ of the group $\mathfrak{S}_{2^{n}}$. Naturally we have the commutative diagram of restriction homomorphisms

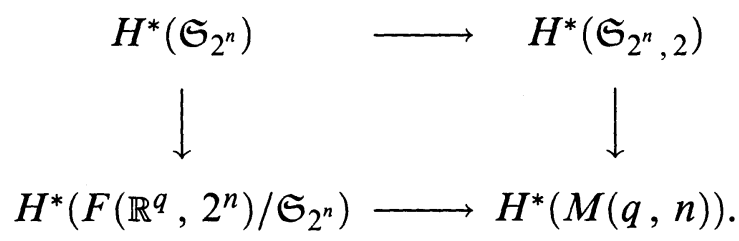

By means of Dickson's and Huỳnh Mùi's invariant theories we shall prove

THEOREM D. The restriction $H^{*}\left(F\left(\mathbb{R}^{q}, 2^{n}\right) / \mathfrak{S}_{2^{n}}\right) \rightarrow H^{*}(M(q, n))$ is a monomorphism.

This is the main step in proving assertion (B) and implying Theorem $\mathrm{C}$ from Theorem A.

The paper is divided into four sections. In $\S 1$ we define the manifold $M(q, n)$ and the embedding $i(q, n): M(q, n) \rightarrow F\left(\mathbb{R}^{q}, 2^{n}\right) / \mathfrak{S}_{2^{n}}$. Section 2 deals with the cohomology of $M(q, n)$ following Steenrod's 
theory on cohomology of wreath products of finite groups. Particularly, we get a geometrical significance of Huynh Mùi's invariants by certain submanifolds of $M(q, n)$. We prove Theorem $\mathrm{D}$ in $\S 3$ and Theorem $\mathrm{C}$ in $\S 4$.

The main results of this paper were announced in [14].

It is a pleasure for me to acknowledge here my deep gratitude to Professor Huỳnh Mùi, from whom I have received an inspiring guidance and a generous help in the proof as well as in the description of the results.

A comment on Theorem C.

R. Wellington [21] computes $P H_{*}\left(\Omega_{0}^{q} S^{q}\right)$. Let $\chi_{i}=Q_{i}[1]^{*}[-2]$, $i>0$, and define Newton polynomials $f_{k}$ by $f_{1}=x_{1}$, and for $k>1, f_{k}=k x_{k}+\sum_{i=1}^{k-1} x_{i} f_{k-i}$.

Let $I=\left(i_{1}, \ldots, i_{k}\right)$ be admissible, that is, $1 \leq i_{1} \leq i_{2} \leq \cdots \leq$ $i_{k}<q . I$ is said to be odd if some $i_{j}$ is odd. Let $\hat{f}_{I}=Q_{J}\left(f_{k}\right)$, with $I=\left(J, i_{k}\right)$. Wellington shows that $\left\{\hat{f}_{I}: I\right.$ is admissible and odd $\}$ forms a basis for $P H_{*}\left(\Omega_{0}^{q} S^{q}\right)$.

Let $\left\{\hat{f}_{i}^{*}\right\}$ be the dual basis of $Q H^{*}\left(\Omega_{0}^{q} S^{q}\right)$.

THEOREM. One can lift the elements $\hat{f}_{I}^{*}$ to $H^{*}\left(\Omega_{0}^{q} S^{q}\right)$, in such a way that

$$
H^{*}\left(\Omega_{0}^{q} S^{q}\right) \cong \mathbb{Z}_{2}\left[\hat{f}_{I}^{*}\right] /\left(\hat{f}_{I}^{*}\right)^{\theta(I)}
$$

where $\theta(I)$ is the smallest integer $2^{m}$ such that $i_{k} 2^{m}>q-1$.

Proof. Let $\xi: H_{*}\left(\Omega_{0}^{q} S^{q}\right) \rightarrow H_{*}\left(\Omega_{0}^{q} S^{q}\right)$ be the Verschiebung or squareroot map, as in [21, p. 26]. Then [21], Lemma 3.5 gives $\xi\left(\chi_{2 i}\right)=\chi_{i}, \xi\left(\chi_{i}\right)=0$

If $i$ is odd; and $\xi(a b)=\xi(a) \xi(b)$. Given $\hat{f}_{I}$, let $y$ be the sum of monomials in the $\chi_{i}$ gotten by multiplying all the subscripts by $2^{m-1}$. Thus $\xi^{m-1} y=\hat{f}_{I}$. In his Lemma 3.5, Wellington shows that there is no $z$ with $\xi^{m}(z)=\hat{f}_{I}$, and the result follows.

1. The wreath products of projective spaces and the configuration spaces. Let $S^{q}, 0 \leq q<\infty$, denote the unit sphere in the Euclid space $\mathbb{R}^{q+1}$ with the base point

$$
*=\underbrace{(1,0, \ldots, 0)}_{q+1} .
$$

We have the canonical base point preserving embeddings

$$
S^{0} \subset S^{1} \subset \cdots \subset S^{q} \subset \cdots .
$$


Set

$$
\left(S^{\infty}, *\right)=\underset{q}{\lim }\left(S^{q}, *\right) .
$$

Let the cyclic group $E$ of order 2 act on $S^{q}$ by the antipodal map. We define the projective space $\mathbb{P}^{q}, 0 \leq q \leq \infty$, by putting

$$
\mathbb{P}^{p}=S^{q} / E \quad \text { for } 0 \leq q<\infty, \quad \mathbb{P}^{\infty}=\underset{q}{\lim } \mathbb{P}^{q} .
$$

The image of the base point $*$ of $S^{q}$ under the canonical projection $S^{q} \rightarrow \mathbb{P}^{q}$ is taken to be that of $\mathbb{P}^{q}$.

Motivated by the Steenrod theory on cohomology of wreath products of finite groups (see Steenrod [20; VIII.3]), we have

1.1. Definition. (i) Let $K$ be a topological space with the base point $*$. Let the group $E$ act on $K \times K$ by permutations of the factors. Then the quotient space $K^{2} \times S^{q}$, where $E$ operates diagonally on $K^{2} \times S^{q}$, is called the wreath product of $K$ by $\mathbb{P}^{q}$ and denoted by $K \int \mathbb{P}^{q}$. The point $[(*, *, *)]$ is considered as the base point of $K \int \mathbb{P}^{q}$.

Obviously, the canonical projection $K \int \mathbb{P}^{q} \rightarrow \mathbb{P}^{q}$ is a splitting (i.e. having a cross section) fibre bundle with fibre $K \times K$.

(ii) Let $q$ be a natural number or $\infty$. We define the $n$-iterated wreath product $M(q, n)$ of projective spaces by induction as follows.

$M(q, 0)=\{*\}$, the space consisting of exactly one point,

$$
M(q, n)=M(q, n-1) \int \mathbb{P}^{q-1}=\mathbb{P}^{q-1} \int \cdots \int \mathbb{P}^{q-1}(n \text { times }) .
$$

Evidently, $M(q, n)$ admits a natural structure of real analytic manifold. It is compact if $q$ is finite. When $q<q^{\prime}$, we have the natural embedding $M(q, n) \subset M\left(q^{\prime}, n\right)$ constructed via the inclusion $S^{q} \subset S^{q^{\prime}}$.

We are going to describe $M(q, n)$ by the other way, which seems to be more useful later. Set

$$
\widetilde{M}(q, 0)=\{*\}, \quad \widetilde{M}(q, n)=\widetilde{M}(q, n-1)^{2} \times S^{q-1} .
$$

The action of $\mathfrak{S}_{2^{n}, 2}=E \int \cdots \int E$ ( $n$ times), the $n$-iterated wreath product of $E$, on $\widetilde{M}(q, n)$ will be defined by induction. Let the group $\mathfrak{S}_{2^{0}, 2}=1$ act trivially on $\widetilde{M}(q, 0)$. Suppose that we are given the action of $\mathfrak{S}_{2^{n-1}, 2}$ on $\widetilde{M}(q, n-1)$, and then that of $\mathfrak{S}_{2^{n}, 2}=$ $\mathfrak{S}_{2^{n-1}, 2} \int E$ on $\widetilde{M}(q, n)$ is defined as follows. Let $x, y \in \widetilde{M}(q, n-1)$, 
$z \in S^{q-1}$; then $(g, h)(x, y, z)=(g x, h y, z)$ for $(g, h) \in \mathfrak{S}_{2^{n-1}, 2}^{2} \subset$ $\mathfrak{S}_{2^{n-1}, 2} \int E=\mathfrak{S}_{2^{n}, 2}, t(x, y, z)=(y, x,-z)$ for $t$ is the generator of $E \subset \mathfrak{S}_{2^{n-1}, 2} \int E$. Notice that this is a free action. Further, we have

$$
M(q, n)=\widetilde{M}(q, n) / \mathfrak{S}_{2^{n}, 2} .
$$

To compute the fundamental group of $M(q, n)$, we need

1.2. Definition. Let $G$ be a group. By the mod 2 wreath product $G \int_{2} \mathbb{Z}$ of $G$ by $\mathbb{Z}$ we mean the semi-direct product $(G \times G) \tilde{\times} \mathbb{Z}$, where the generator of $\mathbb{Z}$ acts on $G \times G$ by permutation of the factors.

1.3. Proposition. We have isomorphisms

$$
\pi_{1}(M(q, n)) \cong \begin{cases}E \int \cdots \int E(n \text { times }), & q>2, \\ \mathbb{Z} \int_{2} \cdots \int_{2} \mathbb{Z}(n \text { times }), & q=2, \\ 1 & q=1 .\end{cases}
$$

Proof. Let $q>1$ and $K$ be an arcwise connected space with base point. Let us consider the homotopy exact sequence of the fibre bundle $K \int \mathbb{P}^{q} \rightarrow \mathbb{P}^{q}:$

$$
\begin{aligned}
& \cdots \rightarrow \pi_{2}\left(\mathbb{P}^{2}\right) \rightarrow \pi_{1}(K \times K) \rightarrow \pi_{1}\left(K \int \mathbb{P}^{q}\right) \rightarrow \pi_{1}\left(\mathbb{P}^{q}\right) \rightarrow \pi_{0}(K \times K) \\
& \ldots \rightarrow \begin{array}{c}
\| 2 \\
1
\end{array} \rightarrow \pi_{1}(K) \times \pi_{1}(K) \rightarrow \pi_{1}\left(K \int \mathbb{P}^{q}\right) \rightarrow \quad \begin{array}{ll}
\| 2 \\
E
\end{array} \rightarrow \quad \begin{array}{l}
\| 2 \\
1 .
\end{array}
\end{aligned}
$$

Since the fibre bundle $K \int \mathbb{P}^{q} \rightarrow \mathbb{P}^{q}$ is splitting, then so is the above exact sequence. Further, via the split, the group $\pi_{1}\left(\mathbb{P}^{q}\right)=E$ operates on the fibre $\pi_{1}(E) \times \pi_{1}(E)$ by permutations of the factors. Hence, we obtain

$$
\pi_{1}\left(K \int \mathbb{P}^{q}\right)=\pi_{1}(K) \int E .
$$

The first part of the proposition follows by induction on $n$. The remaining parts are proved by similar argument using the fact that

$$
\pi_{1}\left(\mathbb{P}^{1}\right) \cong \mathbb{Z}, \quad \pi_{1}\left(\mathbb{P}^{0}\right) \cong 1 .
$$

The proof is completed.

1.4. Proposition. $M(\infty, n)$ and $M(2, n)$ are respectively classifying spaces of the groups $\mathfrak{S}_{2^{n}, 2}$ and $\mathbb{Z} \int_{2} \cdots \int_{2} \mathbb{Z}$ ( $n$ times).

Proof. Note that $K \times K \times S^{q}$ is a covering space over $K \int \mathbb{P}^{q}$. Hence by use of the homotopy exact sequence for the covering we have

$$
\begin{aligned}
\pi_{i}\left(K \int \mathbb{P}^{q}\right) & \cong \pi_{i}\left(K \times K \times S^{q}\right) \\
& \cong \pi_{i}(K) \times \pi_{i}(K) \times \pi_{i}\left(S^{q}\right),
\end{aligned}
$$


for $i>1$. From this, we obtain by induction on $n$ $\pi_{i}(M(q, n)) \cong \pi_{i}\left(S^{q-1}\right) \times \cdots \times \pi_{i}\left(S^{q-1}\right)\left(2^{n}-1\right.$ times $), \quad$ for $i>1$. In particular, we have $\pi_{i}(M(\infty, n))=\pi_{i}(M(2, n))=0$ for $i>1$. From 1.3 and these isomorphisms the proposition follows.

Now is the time to construct the continuous embedding

$$
i(q, n): M(q, n) \rightarrow F\left(\mathbb{R}^{q}, 2^{n}\right) / \mathfrak{S}_{2^{n}} .
$$

To do this, we first define the embedding

$$
\tilde{i}(q, n): \widetilde{M}(q, n) \rightarrow F\left(\mathbb{R}^{q}, 2^{n}\right)
$$

by induction on $n$ as follows. Remember that $S^{q-1}$ is always considered as the unit sphere in $\mathbb{R}^{q}$. We fix a positive constant $\varepsilon<1 / 3$. The map $\tilde{i}(q, 0): \widetilde{M}(q, 0)=\{*\} \rightarrow F\left(\mathbb{R}^{q}, 2^{0}\right)=\mathbb{R}^{q}$ is given by $i(q, 0)(*)=0$. Suppose that the embedding

$$
\begin{aligned}
& \tilde{i}(q, n-1) \\
& \quad=\left(\tilde{i}(q, n-1)_{1}, \ldots, \tilde{i}(q, n-1)_{2^{n-1}}\right): \widetilde{M}(q, n-1) \rightarrow F\left(\mathbb{R}^{q}, 2^{n-1}\right)
\end{aligned}
$$

has been defined, where $\tilde{i}(q, n-1) j$ denotes the $j$ th factor of $\tilde{i}(q, n-1)$. Then we define

$$
\tilde{i}(q, n)=\left(\tilde{i}(q, n)_{1}, \ldots, \tilde{i}(q, n)_{2^{n}}\right): \widetilde{M}(q, n) \rightarrow F\left(\mathbb{R}^{q}, 2^{n}\right)
$$

by the formula

(1.5) $\tilde{i}(q, n)_{j}(x, y, z)= \begin{cases}\varepsilon \tilde{i}(q, n-1)_{j}(x)+z, & j \leq 2^{n-1}, \\ \varepsilon \tilde{i}(q, n-1)_{j-2^{n-1}}(y)-z, & j>2^{n-1},\end{cases}$

for $(x, y, z) \in \widetilde{M}(q, n-1) \times \widetilde{M}(q, n-1) \times S^{q-1}=\widetilde{M}(q, n)$. Since $\varepsilon<1 / 3$, it is easy to show that

$$
\tilde{i}(q, n)_{j}(v) \neq \tilde{i}(q, n)_{k}(v) \text { for } j \neq k, v \in \widetilde{M}(q, n) .
$$

Hence, the map $\tilde{i}(q, n)$ is well defined. We have clearly

1.6. LEMMA. $\tilde{i}(q, n)$ is a continuous injection.

Proof. The continuity of $\tilde{i}(q, n)$ is implied easily from (1.5) by induction on $n$.

To show that $\tilde{i}(q, n)$ is an injection, we note the following simple property, which can also be proved by induction

$$
\sum_{j=1}^{2^{n}} \tilde{i}(q, n)_{j}(v)=0 \text { for } v \in \widetilde{M}(q, n) .
$$


Obviously, $\tilde{i}(q, 0)$ is injective. Suppose that so is $\tilde{i}(q, n-1)$. Let $\left(x_{k}, y_{k}, z_{k}\right) \in \widetilde{M}(q, n)$ for $k=1,2$ such that

$$
\tilde{i}(q, n)\left(x_{1}, y_{1}, z_{1}\right)=\tilde{i}(q, n)\left(x_{2}, y_{2}, z_{2}\right) \text {. }
$$

From this and (1.7) we have

$$
\begin{aligned}
z_{1} & =\frac{1}{2^{n-1}} \sum_{j=1}^{2^{n-1}} \tilde{i}(q, n)_{j}\left(x_{1}, y_{1}, z_{1}\right) \\
& =\frac{1}{2^{n-1}} \sum_{j=1}^{2^{n-1}} \tilde{i}(q, n)_{j}\left(x_{2}, y_{2}, z_{2}\right)=z_{2} .
\end{aligned}
$$

This implies, by (1.5), that

$\tilde{i}(q, n-1)\left(x_{1}\right)=\tilde{i}(q, n-1)\left(x_{2}\right), \quad \tilde{i}(q, n-1)\left(y_{1}\right)=\tilde{i}(q, n-1)\left(y_{2}\right)$.

Using the inductive hypothesis we obtain

$$
x_{1}=x_{2}, \quad y_{1}=y_{2} .
$$

The lemma follows.

Let $q<\infty$. $\tilde{i}(q, n)$ is a continuous injection from the compact space $\widetilde{M}(q, n)$ into the Hausdorff space $F\left(\mathbb{R}^{q}, 2^{n}\right)$; then it is a homeomorphism between its domain and its image. Passing to the direct limit when $q \rightarrow \infty$, we observe that so is $\tilde{i}(\infty, n)$.

$\tilde{i}(q, n) \widetilde{M}(q, n)$ is not $\mathfrak{S}_{2^{n}}$-invariant subspace of $F\left(\mathbb{R}^{q}, 2^{n}\right)$, but so is the following

$$
\mathfrak{S} \widetilde{M}(q, n)=\bigcup_{\sigma \in \mathfrak{S}_{2^{n}}} \sigma \tilde{i}(q, n) \widetilde{M}(q, n) \subset F\left(\mathbb{R}^{q}, 2^{n}\right) .
$$

The map

$$
\begin{array}{ccc}
\widetilde{M}(q, n) / \mathfrak{S}_{2^{n}, 2} & \rightarrow \mathfrak{S} \widetilde{M}(q, n) / \mathfrak{S}_{2^{n}}, \\
{[v]} & \mapsto \quad[\tilde{i}(q, n) v]
\end{array}
$$

is clearly a homeomorphism. It induces the embedding

$$
i(q, n): M(q, n) \rightarrow F\left(\mathbb{R}^{q}, 2^{n}\right) / \mathfrak{S}_{2^{n}} .
$$

When $q=2$, the subspace $i(2, n) M(2, n)$ of $F\left(\mathbb{R}^{2}, 2^{n}\right) / \mathfrak{S}_{2^{n}}$ has been used in Fuks [5] in another formulation.

We have the commutative diagram

$$
\begin{array}{cc}
M\left(q^{\prime}, n\right) \stackrel{i\left(q^{\prime}, n\right)}{\longrightarrow} F\left(\mathbb{R}^{q^{\prime}}, 2^{n}\right) / \mathfrak{S}_{2^{n}} \\
\uparrow & \uparrow \\
M(q, n) \stackrel{i(q, n)}{\longrightarrow} F\left(\mathbb{R}^{q}, 2^{n}\right) / \mathfrak{S}_{2^{n}}
\end{array}
$$


for $q<q^{\prime}$, where the vertical arrows are the natural embeddings.

Remember that, for $q>2, \pi_{1}(M(q, n))=\mathfrak{S}_{2^{n}, 2}$ (Proposition $1.3)$,

$$
\pi_{1}\left(F\left(\mathbb{R}^{q}, 2^{n}\right) / \mathfrak{S}_{2^{n}}\right)=\mathfrak{S}_{2^{n}} \quad(\text { see }[4]) .
$$

Via these isomorphisms, we are going to describe the homomorphism

$$
i_{\#}(q, n): \pi_{1}(M(q, n)) \rightarrow \pi_{1}\left(F\left(\mathbb{R}^{q}, 2^{n}\right) / \mathfrak{S}_{2^{n}}\right), \quad \text { for } q>2 .
$$

Let us consider $\mathfrak{S}_{2^{n}}$ as the symmetric group on (the point set of) the vector space $\mathbb{Z}_{2}^{n}$ of dimension $n$ over $\mathbb{Z}_{2}$. Let $E_{i}, 1 \leq i \leq n$, denote the cyclic group of order 2 generated by the translation defined by the $i$ th unit vector $\varepsilon_{i}$ of $\mathbb{Z}_{2}^{n}$. The map $i_{\#}(q, 0)$ is clearly the identity on the group 1. Suppose that $i_{\#}(q, n-1)$ has been known. Then, by (1.5), the homomorphism

$$
i_{\#}(q, n): \mathfrak{S}_{2^{n}, 2}=\mathfrak{S}_{2^{n-1}, 2} \int E \rightarrow \mathfrak{S}_{2^{n}}
$$

is described as follows.

$$
\begin{aligned}
& \left.i_{\#}(q, n)\right|_{\mathfrak{S}_{2^{n-1}, 2}^{2}}: \mathfrak{S}_{2^{n-1}, 2}^{2} \stackrel{i_{\#}(q, n-1)^{2}}{\rightarrow} \mathfrak{S}_{2^{n-1}}^{2} \stackrel{\subseteq}{\leftrightarrows} \mathfrak{S}_{2^{n}}, \\
& \left.i_{\#}(q, n)\right|_{E}: E \stackrel{\simeq}{\rightrightarrows} E_{n} .
\end{aligned}
$$

So we obtain

$$
i_{\#}(q, n): \mathfrak{S}_{2^{n}, 2} \stackrel{\simeq}{\rightarrow} E_{1} \int E_{2} \cdots \int E_{n} \subset \mathfrak{S}_{2^{n}} .
$$

Via this injection, $\mathfrak{S}_{2^{n}, 2}$ becomes a Sylow 2-subgroup of $\mathfrak{S}_{2^{n}}$. Further, we have the identification

$$
\begin{array}{ccc}
H^{*}\left(\mathfrak{S}_{2^{n}}\right) & \stackrel{\operatorname{Res}\left(\mathfrak{S}_{2^{n}, 2}, \mathfrak{S}_{2^{n}}\right)}{\longrightarrow} & H^{*}\left(\mathfrak{S}_{2^{n}, 2}\right) \\
\| & \| \\
H^{*}\left(F\left(\mathbb{R}^{\infty}, 2^{n}\right) / \mathfrak{S}_{2^{n}}\right) & \stackrel{i^{*}(\infty, n)}{\longrightarrow} & H^{*}(M(\infty, n)) .
\end{array}
$$

As is well known, the restriction $\operatorname{Res}\left(\mathfrak{S}_{2^{n}, 2}, \mathfrak{S}_{2^{n}}\right)$ is a monomorphism. In $\S 3$ we shall prove that so is $i^{*}(q, n)$ for $q$ finite.

2. Cohomology of wreath products of projective spaces. Suppose that the sphere $S^{q}$ is endowed with the following CW-complex structure

$$
S^{q}=\bigcup_{k=0}^{q}\left(e_{k} \cup t e_{k}\right)
$$

where

$$
\begin{aligned}
e_{k} & =\left\{\left(x^{1}, \ldots, x^{k+1}, 0, \ldots, 0\right) \in S^{q} ; x^{k+1}>0\right\} \\
t e_{k} & =\left\{x \in S^{q} ; t x \in e_{k}\right\}
\end{aligned}
$$


$t$ is the generator of $E$. Further, let $K$ be a CW-complex. Then the product CW-structure on $K^{2} \times S^{q}$ is stable under the diagonal action of the group $E$. This induces a CW-structure on $K \int \mathbb{P}^{q}=K^{2} \times_{E} S^{q}$. Denote by $C_{*}(K)$ the chain complex of $K$ with integer coefficients. We have evidently

$$
C_{*}\left(K \int \mathbb{P}^{q}\right)=C_{*}\left(K^{2} \times S^{q}\right) \otimes_{E} \mathbb{Z}=C_{*}(K)^{2} \otimes_{E} C_{*}\left(S^{q}\right) .
$$

In particular, $M(q, n)$ is equipped with the natural CW-complex structure. Further we obtain

$$
C_{*}(M(q, n))=C_{*}(M(q, n-1))^{2} \otimes_{E} C_{*}\left(S^{q}\right) .
$$

Note that, for $q_{1}, q_{2}, \ldots, q_{n}<q$, the complex

$$
M_{q_{1}, \ldots, q_{n}}=\mathbb{P}^{q_{1}} \int \cdots \int \mathbb{P}^{q_{n}}
$$

is a subcomplex of $M(q, n)$.

Now we recall the fundamental result

2.1. TheOREM (Steenrod [20; VIII.3.4], May [8]). Let $K$ be a complex, and $H_{*}(K)$ the vector space over $\mathbb{Z}_{2}$ with the basis $\left\{x_{i} ; i \in I\right\}$, where $I$ is a set ordered linearly. Set $H_{*}\left(\mathbb{P}^{q-1}\right)=\bigoplus_{i=0}^{q-1} \mathbb{Z}_{2} e_{i}$. Then $H_{*}\left(K \int \mathbb{P}^{q-1}\right)$ is the vector space over $\mathbb{Z}_{2}$ with the basis consisting of all elements either of the forms (i), (ii) if $q=\infty$, or of the forms (i), (ii), (iii) if $q<\infty$ as follows.

(i) $x_{i}^{2} \otimes_{E} e_{j}, i \in I, 0 \leq j<q$,

(ii) $x_{i_{1}} \otimes x_{i_{2}} \otimes_{E} e_{0}, i_{1}<i_{2}, i_{1}, i_{2} \in I$,

(iii) $x_{i_{1}} \otimes x_{i_{2}} \otimes_{E}\left(e_{q-1}+t e_{q-1}\right), i_{1}<i_{2}, t_{1}, i_{2} \in I$.

Using this theorem with $M(q, n-1)$ instead of $K$ we may define

2.2. Definition. The Steenrod basis $\operatorname{St}(q, n)$ of the $\mathbb{Z}_{2}$-module $H_{*}(M(q, n))$ is defined by induction on $n$ as follows. Denote by $\operatorname{St}(q, 0)$ the basis of $H_{*}(M(q, 0))=H_{*}(\{*\})=\mathbb{Z}_{2} \cdot 1$ consisting of exactly one vector 1 . Suppose that we have had $\operatorname{St}(q, n-1)=$ $\left\{x_{1}, x_{2}, \ldots\right\}$. Then the basis $\operatorname{St}(q, n)$ consists of all vectors either of the forms (i), (ii) if $q=\infty$, or of the forms (i), (ii), (iii) if $q<\infty$ as follows.

(i) $x_{i}^{2} \otimes_{E} e_{j}, i=1,2, \ldots, 0 \leq j<q$,

(ii) $x_{i_{1}} \otimes x_{i_{2}} \otimes_{E} e_{0}, i_{1}<i_{2}$,

(iii) $x_{i_{1}} \otimes x_{i_{2}} \otimes_{E}\left(e_{q-1}+t e_{q-1}\right), i_{1}<i_{2}$. 
Since the homotopy commutativity of the "multiplication"

$$
\begin{aligned}
\varphi_{n-1}: M(q, n-1) \times M(q, n-1) & \rightarrow M(q, n), \\
(x, y) & \rightarrow[(x, y, *)]
\end{aligned}
$$

it implies that $\operatorname{St}(q, n)$ does not depend on the order given in $\operatorname{St}(q, n-1)$.

$H_{*}(M(q, n))$ contains the "stable" subspace $S(q, n)$ and the "unstable" one $U(q, n)$ with respect to increasing dimension $q$. They are defined by induction as follows.

Set $\underline{S}(q, 0)=\operatorname{St}(q, 0)=\{1\}$. Suppose we are given $\underline{S}(q, n-1)=$ $\left\{y_{1}, y_{2}, \ldots\right\}$. Then $\underline{S}(q, n)$ is the subset of $\operatorname{St}(q, n)$ consisting of all elements of the forms (i), (ii) given in Definition 2.2, in which we put $y_{i}, y_{i_{1}}, y_{i_{2}} \in \underline{S}(q, n-1)$ instead of $x_{i}, x_{i_{1}}, x_{i_{2}}$ respectively. Set $\underline{S}(q, n)=\operatorname{St}(q, n) \backslash \underline{S}(q, n)$. Denote by $S(q, n), U(q, n)$ the vector subspaces of $H_{*}(M(q, n))$ spanned by $\underline{S}(q, n)$ and $\underline{U}(q, n)$ respectively. We have

$$
H_{*}(M(q, n))=S(q, n) \oplus U(q, n) .
$$

Let $i_{*}(M, q): H_{*}(M(q, n)) \rightarrow H_{*}(M(\infty, n))$ be the homomorphism induced by the canonical embedding $i(M, q): M(q, n) \rightarrow$ $M(\infty, n)$. From 2.1 we observe that $i_{*}(M, q) U(q, n)=0$ and $\left.i_{*}(q, n)\right|_{S(q, n)}$ is injective. Further, we have

$$
i_{*}(M, q) S(q, n)=\operatorname{Span}\{x \in \operatorname{St}(\infty, n): h(x)<q\} .
$$

Here the height function $h: \operatorname{St}(\infty, n) \rightarrow \mathbb{Z}$ is defined by induction on $n$ by the formulae

$$
\begin{aligned}
& h(1)=0, \\
& h\left(x_{i}^{2} \otimes_{E} e_{j}\right)=\max \left\{h\left(x_{i}\right), j\right\}, \\
& h\left(x_{i_{1}} \otimes x_{i_{2}} \otimes_{E} e_{0}\right)=\max \left\{h\left(x_{i_{1}}\right), h\left(x_{i_{2}}\right)\right\},
\end{aligned}
$$

for $x_{i}, x_{i_{1}}, x_{i_{2}} \in \mathrm{St}(\infty, n-1)$.

From now on, for each cell complex $K$ we denote its chain complex $C_{*}(K)$ also by $K$.

According to Steenrod [20; V.5.1] we have the $E$-equivariant chain map

$$
\begin{gathered}
d_{q, 1}: \widetilde{M}(q, 1) \rightarrow \widetilde{M}(q, 1) \otimes \widetilde{M}(q, 1), \\
d_{q, 1}\left(e_{k}\right)=\sum_{i=0}^{k}(-1)^{i(k-i)} e_{i} \otimes t^{i} e_{k-i} \quad \text { for } k<q .
\end{gathered}
$$

(Recall that $\widetilde{M}(q, 1)=S^{p-1}$.) 
We obtain the following well-known result (see Nakaoka [12; 3.3] in the case of $q=\infty$ ).

2.5. Proposition. The diagonal approximation $\Delta_{q, n}: M(q, n) \rightarrow$ $M(q, n) \otimes M(q, n)$ is computed by induction as follows.

$$
\begin{aligned}
& \Delta_{q, 1}\left(e_{n}\right)=\sum_{i=0}^{k}(-1)^{i(k-i)} e_{i} \otimes e_{k-i} \quad \text { for } k<q . \\
& \Delta_{q, n}=\tau\left(\Delta_{q, n-1}^{2} \otimes_{E} d_{q, 1}\right) \text { as seen in the diagram } \\
& \begin{array}{cccc}
M(q, n)=M(q, & n-1)^{2} \otimes_{E} \widetilde{M}(q, 1) & \stackrel{\Delta_{q, n}}{\longrightarrow} & M(q, n)^{2} \\
& \downarrow \Delta_{q, n-1}^{2} \otimes_{E} d_{q, 1} & & \| \\
M(q, n-1)^{4} \otimes_{E \times E} & \widetilde{M}(q, 1)^{2} & \stackrel{\tau}{\rightarrow} & \left(M(q, n-1)^{2} \otimes_{E} \widetilde{M}(q, 1)\right)^{2} .
\end{array}
\end{aligned}
$$

Here $\tau$ is the chain map twisting two sets of 3 factors.

Proof. According to Nakaoka $[12 ; 3.3]$ the map $d_{\infty, n}: \widetilde{M}(\infty, n) \rightarrow$ $\widetilde{M}(\infty, n)^{2}$ defined by the inductive formula $d_{\infty, n}=\tau\left(d_{\infty, n-1}^{2} \otimes d_{\infty, 1}\right)$ is an $\mathfrak{S}_{2^{n}, 2}$-equivariant chain map. Hence

$$
d_{q, n}=\left.d_{\infty, n}\right|_{\widetilde{M}(q, n)}: \widetilde{M}(q, n) \rightarrow \widetilde{M}(q, n)^{2}
$$

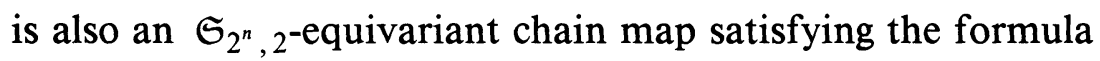

$$
d_{q, n}=\tau\left(d_{q, n-1}^{2} \otimes d_{q, 1}\right)
$$

It is easy to verify that $\widetilde{M}(q, n)$ is a regular $\mathfrak{S}_{2^{n}, 2^{-}}$-free complex and $d_{q, n}$ is carried by the $\mathfrak{S}_{2^{n}, 2}$-equivariant acyclic diagonal carrier $C(\sigma)=\bar{\sigma} \times \bar{\sigma}$, where $\sigma$ denotes a cell of $\widetilde{M}(q, n)$ and $\bar{\sigma}$ is the closure of $\sigma$. Thus $d_{q, n}$ induces the diagonal approximation $\Delta_{q, n}$ of $M(q, n)$ (see e.g. Steenrod [20; V.4.1]). From (2.6) and the definition of $d_{q, 1}$ we get the formulas for computing $\Delta_{q, n}$ given in the proposition. The proof is completed.

We denote by $\mathscr{M}(q, n), \mathscr{M}^{\perp}(q, n)$ the $\mathbb{Z}_{2}$-submodules of $H_{*}(M(q, n))$ generated respectively by $\underline{\mathscr{M}}(q, n)$ and $\underline{\mathscr{M}}^{\perp}(q, n)$, which are defined by induction as follows.

$$
\begin{aligned}
\underline{\mathscr{M}}(q, 0) & =\mathrm{St}(q, 0), \\
\underline{\mathscr{M}}(q, n) & =\left\{x^{2} \otimes_{E} e_{j} ; x \in \underline{\mathscr{M}}(q, n-1), 0 \leq j<q\right\} \subset \operatorname{St}(q, n), \\
\underline{\mathscr{M}}^{\perp}(q, n) & =\operatorname{St}(q, n) \backslash \underline{\mathscr{M}}(q, n) .
\end{aligned}
$$

So we get

$$
H_{*}(M(q, n))=\mathscr{M}(q, n) \oplus \mathscr{M}^{\perp}(q, n) .
$$


Define by induction for $q_{1}, \ldots, q_{n}<q$

$$
m_{q_{1}, \ldots, q_{n}}=m_{q_{1}, \ldots, q_{n-1}}^{2} \otimes_{E} e_{q_{n}} .
$$

It is easily seen that $m_{q_{1}}, \ldots, q_{n}$ is the unique cell of highest dimension in the subcomplex $M_{q_{1}, \ldots, q_{n}}=\mathbb{P}^{q_{1}} \int \cdots \int \mathbb{P}^{q_{n}}$ of $M(q, n)$. Thus $m_{q_{1}, \ldots, q_{n}}$ is the fundamental class of the submanifold $M_{q_{1}, \ldots, q_{n}}$ in the manifold $M(q, n)$. Obviously we have

$$
\underline{\mathscr{M}}(q, n)=\left\{m_{q_{1}, \ldots, q_{n}} ; 0 \leq q_{1}, \ldots, q_{n}<q\right\} .
$$

By a simple computation based on Proposition 2.5 we obtain easily

2.7. Lemma. The comultiplication $\Delta$ of the coalgebra $H_{*}(M(q, n))$ satisfies the formulas

$$
\begin{aligned}
& \Delta\left(m_{q_{1}, \ldots, q_{n}}\right)= \sum_{r_{i}+s_{i}=q_{i}} m_{r_{1}, \ldots, r_{n}} \otimes m_{s_{1}, \ldots, s_{n}} \\
& \text { for } 0 \leq q_{i}, \quad r_{i}, \quad s_{i}<q, \quad 1 \leq i \leq n . \\
& \Delta \mathscr{M}^{\perp}(q, n) \subset H_{*}(M(q, n)) \otimes \mathscr{M}^{\perp}(q, h) \\
&+\mathscr{M}^{\perp}(q, n) \otimes H_{*}(M(q, n)) .
\end{aligned}
$$

As a consequence, we have the following proposition essentially due to Steenrod [20], Nakaoka [12].

2.8. Proposition (cf. [6], [12]).

$$
H^{*}(M(q, n))=\mathscr{M}^{\perp}(q, n)^{*} \oplus \mathscr{M}(q, n)^{*},
$$

where $\mathscr{M}^{\perp}(q, n)^{*}$ is an ideal and $\mathscr{M}(q, n)^{*}$ is a subalgebra. Here * denotes the dual defined by the Steenrod basis.

Now we study the structure of the algebra $\mathscr{M}(q, n)^{*}$. Set

$$
\bar{V}_{n, s}=m_{n-s}^{m_{0}^{*, \ldots, 0},} \underbrace{1,0, \ldots, 0}_{s} \in H^{*}(M(q, n)), \quad \text { for } 1 \leq s \leq n, q \geq 1 \text {. }
$$

According to Lemma 2.7 we have in $H^{*}(M(q, n))$

$$
m_{q_{1}, \ldots, q_{n}}^{*}=\bar{V}_{n, 1}^{q_{n}} \ldots \bar{V}_{n, n}^{q_{1}},
$$

for $q_{1}, \ldots, q_{n}<q$. Clearly, the algebra $\mathscr{M}(\infty, n)^{*}$ has the following simple structure

$$
\mathscr{M}(\infty, n)^{*}=\mathbb{Z}_{2}\left[\bar{V}_{n, 1}, \ldots, \bar{V}_{n, n}\right]
$$


(Compare with Nakaoka [12; 4.2].) The natural embedding $M(q, n) \subset$ $M(\infty, n)$ induces the surjection of algebras $\mathscr{M}(\infty, n)^{*} \rightarrow \mathscr{M}(q, n)^{*}$, whose kernel is

$$
\operatorname{Span}\left\{m_{q_{1}, \ldots, q_{n}}^{*}: h\left(m_{q_{1}, \ldots, q_{n}}\right)=\max \left\{q_{1}, \ldots, q_{n}\right\} \geq q\right\} .
$$

This coincides with the ideal

$$
\left(\bar{V}_{n, 1}^{q}, \ldots, \bar{V}_{n, n}^{q}\right) \quad \text { of } \quad \mathbb{Z}_{2}\left[\bar{V}_{n, 1}, \ldots, \bar{V}_{n, n}\right] .
$$

By the above discussion, we get

2.10. THEOREM. There is an isomorphism of algebras

$$
\mathscr{M}(q, n)^{*} \cong \mathbb{Z}_{2}\left[\bar{V}_{n, 1}, \ldots, \bar{V}_{n, n}\right] /\left(\bar{V}_{n, 1}^{q}, \ldots, \bar{V}_{n, n}^{q}\right) .
$$

For latter use, we recall Huỳnh Mùi's result on the homomorphism

$$
H^{*}(M(\infty, n)) \rightarrow H^{*}\left(\mathbb{P}_{1}^{\infty} \times \cdots \times \mathbb{P}_{n}^{\infty}\right)
$$

Let $\mathbb{P}_{k}^{\infty}$, for $k=1, \ldots, n$, be the real projective space of infinite dimension. It is easily seen that

$$
\mathbb{P}_{1}^{\infty} \times \cdots \times \mathbb{P}_{n}^{\infty}=B E^{n} .
$$

$\mathbb{P}_{k}^{\infty}$ has been equipped with the $\mathrm{CW}$-complex structure as noted at the beginning of $\S 2$. It has exactly one cell $e_{i}^{k}$ in each dimension $i$, for $0 \leq i<\infty$. Further, we have

$$
H_{*}\left(\mathbb{P}_{k}^{\infty}\right)=\bigoplus_{i=0}^{\infty} \mathbb{Z}_{2} \cdot e_{i}^{k}
$$

Let $y_{k} \in H^{*}\left(\mathbb{P}_{k}^{\infty}\right)$ be the dual element of $e_{1}^{k} \in H_{*}\left(\mathbb{P}_{k}^{\infty}\right)$ via the basis $\left\{e_{i}^{k} ; 0 \leq i<\infty\right\}$. As it is well known, we get

$$
H^{*}\left(E^{n}\right)=H^{*}\left(\mathbb{P}_{1}^{\infty} \times \cdots \times \mathbb{P}_{n}^{\infty}\right)=\mathbb{Z}_{2}\left[y_{1}, \ldots, y_{n}\right] .
$$

We define the injection $i_{n}: E^{n} \rightarrow \mathfrak{S}_{2^{n}, 2}$ by induction as follows.

$$
\begin{aligned}
i_{1}=\mathrm{id}_{E^{1}}: E^{1} & \rightarrow \mathfrak{S}_{2,2}=E^{1}, \\
i_{n}: E^{n}=E^{n-1} \times E & \rightarrow \mathfrak{S}_{2^{n}, 2}=\mathfrak{S}_{2^{n-1}, 2} \int E, \\
(a, b) & \mapsto\left(i_{n-1} a, i_{n-1} a ; b\right),
\end{aligned}
$$

for $a \in E^{n-1}, b \in E$. 
The homomorphism $i_{n}$ induces the homotopy class of the map of classifying spaces $d_{n}: \mathbb{P}_{1}^{\infty} \times \cdots \times \mathbb{P}_{n}^{\infty} \rightarrow M(\infty, n)$ which is constructed also by induction.

$$
\begin{aligned}
d_{1}=\mathrm{id}: \mathbb{P}_{1}^{\infty} & \rightarrow M(\infty, 1)=\mathbb{P}_{1}^{\infty}, \\
d_{n}: \mathbb{P}_{1}^{\infty} \times \cdots \times \mathbb{P}_{n}^{\infty} & \rightarrow M(\infty, n)=M(\infty, n-1) \int \mathbb{P}_{n}^{\infty}, \\
(a, b) & \mapsto\left(d_{n-1} a, d_{n-1} a ; b\right),
\end{aligned}
$$

for $a \in \mathbb{P}_{1}^{\infty} \times \cdots \times \mathbb{P}_{n-1}^{\infty}, b \in \mathbb{P}_{n}^{\infty}$. So we obtain the identification

$$
\begin{array}{ccc}
H^{*}\left(\mathfrak{S}_{2^{n}, 2}\right) & \stackrel{\operatorname{Res}\left(E^{n}, \mathfrak{S}_{2^{n}, 2}\right)}{\longrightarrow} & H^{*}\left(E^{n}\right) \\
H^{*}(M(\infty, n)) & \stackrel{d_{n}^{*}}{\longrightarrow} & H^{*}\left(\mathbb{P}_{1}^{\infty} \times \cdots \times \mathbb{P}_{n}^{\infty}\right) .
\end{array}
$$

Under this, we set

$$
V_{n, s}=d_{n}^{*} \bar{V}_{n, s}=\operatorname{Res}\left(E^{n}, \mathfrak{S}_{2^{n}, 2}\right) \bar{V}_{n, s}, \quad 1 \leq s \leq n .
$$

The following allows us to compute $V_{n, s}$ by induction.

2.11. Proposition. Let $d^{*}=\operatorname{Res}\left(E^{n}, E^{n-1} \int E\right)$, and let $P: H^{*}\left(E^{n-1}\right) \rightarrow H^{*}\left(E^{n-1} \int E\right)$ be the Steenrod map (see [20; VII.2]). Then we have

$$
d_{n}^{*} m_{q_{1}, \ldots, q_{n-1}, 0}^{*}=d^{*} P d_{n-1}^{*} m_{q_{1}, \ldots, q_{n-1}}^{*} .
$$

Particularly, we get $V_{n, s}=d^{*} P V_{n-1, s-1}$ for $1<s \leq n$. Meanwhile $V_{n, 1}=y_{n}$.

Proof. We recall that

$$
m_{q_{1}, \ldots, q_{n-1}, 0}=m_{q_{1}, \ldots, q_{n-1}}^{2} \otimes_{E} e_{0} .
$$

Passing it to the dual, from the definition of the map $P$, we obtain

$$
m_{q_{1}, \ldots, q_{n-1}, 0}^{*}=P m_{q_{1}, \ldots, q_{n-1}}^{*} .
$$

On the other hand, we have the commutative diagram

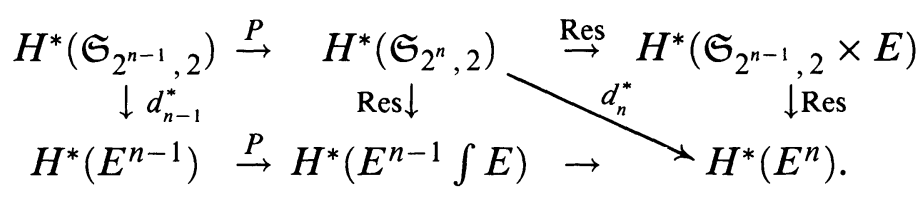

So we get

$$
d_{n}^{*} m_{q_{1}, \ldots, q_{n-1}, 0}^{*}=d_{n}^{*} P m_{q_{1}, \ldots, q_{n-1}}^{*}=d^{*} P d_{n-1}^{*} m_{q_{1}, \ldots, q_{n-1}}^{*} .
$$


Apply this formula with

$$
\left(q_{1}, \ldots, q_{n-1}, 0\right)=(\underbrace{0, \ldots, 0}_{n-s}, \underbrace{1,0, \ldots, 0}_{s})
$$

to obtain

$$
V_{n, s}=d^{*} P V_{n-1, s-1} \text { for } 1<s \leq n .
$$

At last, we note that

$$
m_{\underbrace{0, \ldots, 0,1}_{n}}^{0, m^{2}, \ldots, 0} \otimes e_{1}^{0,1}=1^{2} \otimes_{E} e_{1} .
$$

Hence, we have

$$
m_{n}^{m_{0, \ldots, 0,1}^{*}}=P(1) \otimes y_{n} .
$$

Since $d_{n}^{*}$ is a homomorphism of $H^{*}(E)$-modules, we obtain

$$
V_{n, 1}=d_{n}^{*} \underbrace{m_{0, \ldots, 0,1}^{*}}_{n}=d_{n}^{*}\left(P(1) \otimes y_{n}\right)=y_{n} .
$$

This completes the proof.

In principle, Proposition 2.11 allows us to determine completely $V_{n, s}$ as a polynomial of $y_{1}, \ldots, y_{n}$. But, it is rather difficult to realize the direct computation. To overcome this difficulty, we need the following well-known fact. Let $G$ be a finite group and $S$ a subgroup of $G$. The Weyl group $W_{G}(S)=N_{G}(S) / C_{G}(S)$ of $S$ in $G$ operates on $H^{*}(S)$ by the adjoint isomorphisms. We have (see e.g. Steenrod $[20 ;$ V.7])

$$
\operatorname{Im}\left[\operatorname{Res}(S, G): H^{*}(G) \rightarrow H^{*}(S)\right] \subset H^{*}(S)^{W_{G}(S)} .
$$

In [6; II.5] Huỳnh Mùi showed that

$$
\begin{aligned}
& W=W_{\mathfrak{S}_{2^{n}, 2}}\left(E^{n}\right)=\mathrm{GL}_{n, 2}, \\
& H^{*}\left(E^{n}\right)^{W}=\mathbb{Z}_{2}\left[y_{1}, \ldots, y_{n}\right]^{\mathrm{GL}_{n, 2} .}
\end{aligned}
$$

Here $\mathrm{GL}_{n, 2}$ denotes the subgroup of $\mathrm{GL}\left(n ; \mathbb{Z}_{2}\right)$ consisting of all lower triangular matrices with 1 in the diagonal entries, and the group $\mathrm{GL}\left(n, \mathbb{Z}_{2}\right)$ acts canonically on $\mathbb{Z}_{2}\left[y_{1}, \ldots, y_{n}\right]$.

Note that $V_{n, s}$ is an invariant under the action of $\mathrm{GL}_{n, 2}$ (since $\left.V_{n, s} \in \operatorname{Im} \operatorname{Res}\left(E^{n}, \mathfrak{S}_{2^{n}, 2}\right)\right)$ and contains $y_{n-s+1}$ as a factor (according to 2.11). So we obtain (see Huỳnh Mùi [6; II.5.4])

$$
V_{n, s}=\prod_{\lambda_{1} \in \mathbb{Z}_{2}}\left(\lambda_{1} y_{n}+\cdots+\lambda_{s-1} y_{n-s+2}+y_{n-s+1}\right) \text {. }
$$


This implies directly that $V_{n, 1}, \ldots, V_{n, s}$ are algebraically independent (see also [6; I.3.4]).

2.15. Definition. The injection of modules $\alpha: \mathbb{Z}_{2}\left[V_{n, 1}, \ldots, V_{n, n}\right]$ $\rightarrow H^{*}\left(\mathfrak{S}_{2^{n}, 2}\right)$ is defined by

$$
\alpha\left(V_{n, 1}^{q_{n}} \ldots V_{n, n}^{q_{1}}\right)=m_{q_{1}, \ldots, q_{n}}^{*}
$$

From (2.9), $\alpha$ is a homomorphism of graded algebras.

2.16. Proposition (Huỳnh Mùi [6; II.5.2]). We have the exact sequence of algebras which is splitting via the homomorphism $\alpha$

$$
0 \rightarrow \mathscr{M}^{\perp}(\infty, n)^{*} \stackrel{j}{\rightarrow} H^{*}\left(\mathfrak{S}_{2^{n}, 2}\right) \stackrel{d_{n}^{*}}{\rightarrow} \mathbb{Z}_{2}\left[V_{n, 1}, \ldots, V_{n, n}\right] \rightarrow 0,
$$

where $j$ denotes the natural embedding.

3. The monomorphisms $i^{*}(q, n)$. The purpose of this section is to prove

3.1. THEOREM. $i^{*}(q, n): H^{*}\left(F\left(\mathbb{R}^{q}, 2^{n}\right) / \mathfrak{S}_{2^{n}}\right) \rightarrow H^{*}(M(q, n))$ is a monomorphism for $q \geq 1, n \geq 0$.

At first, we recall some things about the (Hopf) algebra

$$
H_{*}\left(F\left(\mathbb{R}^{q}, \infty\right) / \mathfrak{S}_{\infty}\right) .
$$

For a finite positive integer $q$, we define the embeddings

$$
\begin{aligned}
l_{m}: F\left(\mathbb{R}^{q}, m\right) / \mathfrak{S}_{m} & \rightarrow F\left(\mathbb{R}^{q}, m+1\right) / \mathfrak{S}_{m+1}, \\
{\left[\left(x_{1}, \ldots, x_{m}\right)\right] } & \mapsto\left[\left(x_{1}, \ldots, x_{m}, x\right)\right],
\end{aligned}
$$

where

$$
x=\frac{1}{m} \sum_{i=1}^{m} x_{i}-(\underbrace{R+1,0, \ldots, 0}_{q}), \quad R=\max _{k}\left\|x_{k}-\frac{1}{m} \sum_{i=1}^{m} x_{i}\right\| .
$$

Here and in what follows, $\|\cdot\|$ denotes the Euclid metric in $\mathbb{R}^{q}$. (Compare with Fuks [5], when $q=2$.) Via these embeddings, we set

$$
F\left(\mathbb{R}^{q}, \infty\right) / \mathfrak{S}_{\infty}=\underset{m}{\lim } F\left(\mathbb{R}^{q}, m\right) / \mathfrak{S}_{m}
$$

Let us consider in this space a product induced by the maps

$$
\begin{gathered}
\mu_{m, n}: F\left(\mathbb{R}^{q}, m\right) / \mathfrak{S}_{m} \times F\left(\mathbb{R}^{q}, n\right) / \mathfrak{S}_{n} \rightarrow F\left(\mathbb{R}^{q}, m+n\right) / \mathfrak{S}_{m+n} \\
{\left[\left(x_{1}, \ldots, x_{m}\right)\right] \times\left[\left(y_{1}, \ldots, y_{n}\right)\right]} \\
\mapsto\left[\left(x_{1}, \ldots, x_{m}, y_{1}+z, \ldots, y_{n}+z\right)\right]
\end{gathered}
$$


where

$$
\begin{gathered}
z=\frac{1}{m} \sum_{i=1}^{m} x_{i}-\frac{1}{n} \sum_{j=1}^{n} y_{i}-(R_{1} \underbrace{+R_{2}+1,0, \ldots, 0}_{q}), \\
R_{1}=\max _{k}\left\|x_{k}-\frac{1}{m} \sum_{i=1}^{m} x_{i}\right\|, \quad R_{2}=\max _{k}\left\|y_{k}-\frac{1}{n} \sum_{j=1}^{n} y_{i}\right\| .
\end{gathered}
$$

(Compare to Fuks [5] when $q=2$.)

$F\left(\mathbb{R}^{q}, \infty\right) / \mathfrak{S}_{\infty}$ equipped with this product is not an $H$-space. However, $H_{*}\left(F\left(\mathbb{R}^{q}, \infty\right) / \mathfrak{S}_{\infty}\right)$ is a Hopf algebra (see $\left.[10, \S 5]\right)$. The direct limits when $q \rightarrow \infty$ of the systems of map (3.2), (3.3) belong to the homotopy classes of maps on classifying spaces induced respectively by the canonical injections

$$
\mathfrak{S}_{m} \rightarrow \mathfrak{S}_{m+1}, \quad \mathfrak{S}_{m} \times \mathfrak{S}_{n} \rightarrow \mathfrak{S}_{m+n} .
$$

Hence, $H_{*}\left(F\left(\mathbb{R}^{q}, \infty\right) / \mathfrak{S}_{\infty}\right)$ is a Hopf subalgebra of the Hopf algebra $H_{*}\left(F\left(\mathbb{R}^{\infty}, \infty\right) / \mathfrak{S}_{\infty}\right)=H_{*}\left(\mathfrak{S}_{\infty}\right)$ introduced by Nakaoka in [12, §2]. The structure of this Hopf algebra will be studied in $\S 4$.

Besides this, the algebra $H_{*}\left(F\left(\mathbb{R}^{q}, \infty\right) / \mathfrak{S}_{\infty}\right)$ is equipped with the multiplicity such that

$$
{ }_{m} H_{*}\left(F\left(\mathbb{R}^{q}, \infty\right) / \mathfrak{S}_{\infty}\right)=H_{*}\left(F\left(\mathbb{R}^{q}, m\right) / \mathfrak{S}_{m}, F\left(\mathbb{R}^{q}, m-1\right) / \mathfrak{S}_{m-1}\right)
$$

(see e.g. Nguyên H. V. Hung [15, §2]). The reader who is not familiar with the notion of "algebra with multiplicity" can refer to T. Nakamura [11]. For such an algebra $A=\bigoplus_{n \geq 0} A$, we set

$$
A(m)=\bigoplus_{n \leq m} A \text {. }
$$

Particularly, we have

$$
H_{*}\left(F\left(\mathbb{R}^{q}, \infty\right) / \mathfrak{S}_{\infty}\right)(m)=H_{*}\left(F\left(\mathbb{R}^{q}, m\right) / \mathfrak{S}_{m}\right) .
$$

Let

$$
N_{k_{0}, \ldots, k_{n-1}} \in H_{*}\left(F\left(\mathbb{R}^{q}, \infty\right) / \mathfrak{S}_{\infty}\right), \quad k_{0}, \ldots, k_{n-1} \geq 0,
$$

be the Nakamura elements $[15 ; \S 2]$. These are of multiplicity $2^{n}$. Then we have (see $[15 ; 2.15])$.

3.4. THeOREM (Nakamura [11], May [10], Huỳnh Mùi [7]).

(i) Let $q>0$ and

$$
J^{+}(q)=\left\{K=\left(k_{0}, \ldots, k_{n-1}\right) ; n>0, k_{0}>0, k_{i} \in \mathbb{Z}_{+}, \sum_{i=0}^{n-1} k_{i}<q\right\} \text {. }
$$


Then $H_{*}\left(F\left(\mathbb{R}^{q}, \infty\right) / \mathfrak{S}_{\infty}\right)=\mathbb{Z}_{2}\left[N_{K} ; K \in J^{+}(q)\right]$ as algebras with multiplicity. So we have $H_{*}\left(F\left(\mathbb{R}^{q}, m\right) / \mathfrak{S}_{m}\right)=\mathbb{Z}_{2}\left[N_{K} ; K \in J^{+}(q)\right](m)$, for $0 \leq m \leq \infty$. In other words, $H_{*}\left(F\left(\mathbb{R}^{q}, m\right) / \mathfrak{S}_{m}\right)$ has the $\mathbb{Z}_{2}$-basis consisting of all monomials in $\mathbb{Z}_{2}\left[N_{K} ; K \in J^{+}(q)\right]$ of multiplicities $\leq m$. This is called the Nakamura basis.

(ii) The homomorphism

$$
i_{*}(F, q): H_{*}\left(F\left(\mathbb{R}^{q}, \infty\right) / \mathfrak{S}_{\infty}\right) \rightarrow H_{*}\left(F\left(\mathbb{R}^{\infty}, \infty\right) / \mathfrak{S}_{\infty}\right)
$$

induced by the canonical embeddings $F\left(\mathbb{R}^{q}, m\right) \subset F\left(\mathbb{R}^{\infty}, m\right), 0 \leq$ $m<\infty$, is an injection. It sends $N_{K}$ to the element denoted by the same notation $N_{K}$ for $K \in J^{+}(q)$.

On the other hand, let $\rho_{2^{n}}: \mathfrak{S}_{2^{n}} \rightarrow O\left(2^{n}\right)$ denote the natural representation of the symmetric group $\mathfrak{S}_{2^{n}}$ in the orthogonal group $O\left(2^{n}\right)$. As is well known:

$$
H^{*}\left(O\left(2^{n}\right)\right)=\mathbb{Z}_{2}\left[W_{1}, \ldots, W_{2^{n}}\right],
$$

where $W_{i}$ denotes the $i$ th universal Stiefel-Whitney class (of dimension $i)$. We define the $\left(2^{n}-2^{s}\right)$ th Stiefel-Whitney class of $\rho_{2^{n}}$ by putting

$$
W_{n, s}=\rho_{2^{n}}^{*}\left(W_{2^{n}-2^{s}}\right) \in H^{*}\left(\mathfrak{S}_{2^{n}}\right), \quad 0 \leq s<n .
$$

Further, we set

$$
\bar{Q}_{n, s}=\operatorname{Res}\left(\mathfrak{S}_{2^{n}, 2}, \mathfrak{S}_{2^{n}}\right) W_{n, s} \in H^{*}\left(\mathfrak{S}_{2^{n}, 2}\right), \quad 0 \leq s<n .
$$

3.5. Proposition. Let

$$
\begin{aligned}
& i(M, q): M(q, n) \rightarrow M(\infty, n), \\
& i(\infty, n): M(\infty, n) \rightarrow F\left(\mathbb{R}^{\infty}, 2^{n}\right) / \mathfrak{S}_{2^{n}}
\end{aligned}
$$

be the well-known embeddings. Then we have

$$
\begin{aligned}
& i^{*}(M, q) i^{*}(\infty, n) \operatorname{Ker} \operatorname{Res}\left(\mathfrak{S}_{2^{n-1}}^{2}, \mathfrak{S}_{2^{n}}\right) \\
& \quad=\bar{Q}_{n, 0} \mathbb{Z}_{2}\left[\bar{Q}_{n, 0}, \ldots, \bar{Q}_{n, n-1}\right] / I(\bar{Q}, q) .
\end{aligned}
$$

Here $I(\bar{Q}, q)$ denotes the ideal of $\bar{Q}_{n, 0} \mathbb{Z}_{2}\left[\bar{Q}_{n, 0}, \ldots, \bar{Q}_{n, n-1}\right]$ generated by monomials of degree $q$.

The proof of this proposition will be given in the end of the section. Now using this result we prove the main theorem of this section.

Proof of Theorem 3.1. The proof proceeds by induction on $n$. Obviously, $i^{*}(q, 0): H^{*}\left(\mathbb{R}^{q}\right) \rightarrow H^{*}(\{*\})$ is a monomorphism. Suppose 
that so is $i^{*}(q, n-1)$. By means of (2.3), (3.3) and the definition of $i(q, n)$ we have the homotopy commutative diagram

$$
\begin{aligned}
& F\left(\mathbb{R}^{q}, 2^{n}\right) / \mathfrak{S}_{2^{n}} \stackrel{\mu=\mu_{2^{n-1}, 2^{n-1}}}{\longleftarrow}\left(F\left(\mathbb{R}^{q}, 2^{n-1}\right) / \mathfrak{S}_{2^{n-1}}\right)^{2} \\
& \uparrow i(q, n) \quad \uparrow l(q, n-1)^{2} \\
& M(q, n) \stackrel{\varphi=\varphi_{n-1}}{\longleftarrow} \quad M(q, n-1)^{2} .
\end{aligned}
$$

So we get the commutative diagram in which each row is exact (according to 2.1 and 3.4 )

$$
\begin{aligned}
& 0 \rightarrow \quad \operatorname{Ker} \mu^{*} \quad \rightarrow H^{*}\left(F\left(\mathbb{R}^{q}, 2^{n}\right) / \mathfrak{S}_{2^{n}} \stackrel{\mu^{*}}{\rightarrow} H^{*}\left(F\left(\mathbb{R}^{q}, 2^{n-1}\right) / \mathfrak{S}_{2^{n-1}}\right)^{2} \rightarrow 0\right. \\
& \begin{aligned}
& \downarrow i^{*}(q, n) & & \downarrow i^{*}(q, n) & & \downarrow \\
0 \rightarrow \quad \operatorname{Ker} \varphi^{*} & \rightarrow & H^{*}(M(q, n)) & \stackrel{\varphi^{*}}{\rightarrow} & H^{*}(M(q, n, n-1))^{2} & \rightarrow 0 .
\end{aligned}
\end{aligned}
$$

By the inductive hypothesis,

$$
i^{*}(q, n-1)^{2}=i^{*}(q, n-1) \otimes i^{*}(q, n-1)
$$

is an injection. According to the 5-lemma, to prove Theorem 3.1, we need only to show that $\left.i^{*}(q, n)\right|_{\operatorname{Ker} \mu^{*}}: \operatorname{Ker} \mu^{*} \rightarrow \operatorname{Ker} \varphi^{*}$ is a monomorphism.

Let us consider the commutative diagram

$$
\begin{array}{ccc}
H^{*}\left(F\left(\mathbb{R}^{\infty}, 2^{n}\right) / \mathfrak{S}_{2^{n}}\right) \stackrel{\operatorname{Res}\left(\mathfrak{S}_{2^{n-1}}^{2}, \mathfrak{S}_{2^{n}}\right)}{\longrightarrow} & H^{*}\left(F\left(\mathbb{R}^{\infty}, 2^{n-1}\right) / \mathfrak{S}_{2^{n-1}}\right)^{2} \\
\downarrow^{*}(F, q) & & \\
H^{*}\left(F\left(\mathbb{R}^{q}, 2^{n}\right) / \mathfrak{S}_{2^{n}}\right) & \stackrel{\mu^{*}}{\longrightarrow} & H^{*}\left(F\left(\mathbb{R}^{q}, 2^{n-1}\right) / \mathfrak{S}_{2^{n-1}}\right)^{2},
\end{array}
$$

where the vertical arrows are induced from the canonical embeddings $F\left(\mathbb{R}^{q}, m\right) \rightarrow F\left(\mathbb{R}^{\infty}, m\right)$ for $m=2^{n-1}, 2^{n}$. By means of Theorem 3.4 and the definition of the algebra structure on $H_{*}\left(F\left(\mathbb{R}^{q}, \infty\right) / \mathfrak{S}_{\infty}\right)$ we have

$$
\begin{aligned}
& \operatorname{Ker} \operatorname{Res}\left(\mathfrak{S}_{2^{n-1}}^{2}, \mathfrak{S}_{2^{n}}\right)=\operatorname{Span}\left\{N_{k_{0}, \ldots, k_{n-1}}^{*} ; k_{0}>0\right\}, \\
& \begin{aligned}
\operatorname{Ker} \mu^{*} & =\operatorname{Span}\left\{N_{k_{0}, \ldots, k_{n-1}}^{*} ;\left(k_{0}, \ldots, k_{n-1}\right) \in J^{+}(q)\right\} \\
& =i^{*}(F, q) \operatorname{Span}\left\{N_{k_{0}, \ldots, k_{n-1}}^{*} ; k_{0}>0\right\} \\
& =i^{*}(F, q) \operatorname{Ker} \operatorname{Res}\left(\mathfrak{S}_{2^{n-1}}^{2}, \mathfrak{S}_{2^{n}}\right) .
\end{aligned}
\end{aligned}
$$


From Proposition 3.5 and the commutative diagram

$$
\begin{aligned}
& H^{*}\left(F\left(\mathbb{R}^{\infty}, 2^{n}\right) / \mathfrak{S}_{2^{n}}\right) \stackrel{i^{*}(\infty, n)}{\longrightarrow} H^{*}(M(\infty, n)) \\
& \begin{array}{crr} 
& & \\
i^{*}(F, q) & & i^{*}(M, q) \\
H^{*}\left(F\left(\mathbb{R}^{q}, 2^{n}\right) / \mathfrak{S}_{2^{n}}\right) \stackrel{i^{*}(q, n)}{\longrightarrow} & H^{*}(M(q, n))
\end{array}
\end{aligned}
$$

it implies that the following is an epimorphism

$$
\left.i^{*}(q, n)\right|_{\operatorname{Ker} \mu^{*}}: \operatorname{Ker} \mu^{*} \rightarrow \bar{Q}_{n, 0} \mathbb{Z}_{2}\left[\bar{Q}_{n, 0}, \ldots, \bar{Q}_{n, n-1}\right] / I(\bar{Q}, q) .
$$

Note that the domain and the image of this epimorphism are isomorphic to each other as graded modules of finite type over $\mathbb{Z}_{2}$ via the formal correspondence

$$
N_{k_{0}, \ldots, k_{n-1}}^{*} \leftrightarrow Q_{n, 0}^{k_{0}} \cdots Q_{n, 1-1}^{k_{n-1}} \text { for } k_{0}>0, \quad \sum_{i=0}^{n-1} k_{i}<q .
$$

Hence, the above epimorphism is an isomorphism. As a consequence, $\left.i^{*}(q, n)\right|_{\operatorname{Ker} \mu^{*}}: \operatorname{Ker} \mu^{*} \rightarrow \operatorname{Ker} \varphi^{*}$ is a monomorphism. Theorem 3.1 is proved.

The remaining part of this section is devoted to prove Proposition 3.5.

Let $G$ be a finite group, and $E(G)$ a set of representatives for the conjugacy classes of maximal elementary abelian 2-subgroups of $G$. We recall

3.8. Proposition (Quillen [17]). Let $G=\mathfrak{S}_{m}$ or $\mathfrak{S}_{m, 2}$, the Sylow 2-subgroup of $\mathfrak{S}_{m}$. Then the homomorphism

$$
\text { Res: } H^{*}(G) \rightarrow \prod_{A \in E(G)} H^{*}(A)
$$

given by the restrictions $\operatorname{Res}(A, G): H^{*}(G) \rightarrow H^{*}(A)$ for $A \in E(G)$ is a monomorphism.

In [6; II.6.2], Huỳnh Mùi has computed the image of $\operatorname{Res}\left(A, \mathfrak{S}_{m}\right)$ for $m=2^{n}$ and $A=E^{n}$ by means of Dickson's invariant theory. Namely, he has shown that

$$
W_{\mathfrak{S}_{2^{n}}}\left(E^{n}\right) \cong \mathrm{GL}\left(n, \mathbb{Z}_{2}\right),
$$

$\operatorname{Im} \operatorname{Res}\left(E^{n}, \mathfrak{S}_{2^{n}}\right)=H^{*}\left(E^{n}\right)^{W_{\mathfrak{S}_{2^{n}}}\left(E^{n}\right)}=\mathbb{Z}_{2}\left[y_{1}, \ldots, y_{n}\right]^{\mathrm{GL}\left(n, \mathbb{Z}_{2}\right)}$. 
Here the Weyl group $W_{\mathfrak{S}_{2^{n}}}\left(E^{n}\right)$ of $E^{n}$ in $\mathfrak{S}_{2^{n}}$ acts on $H^{*}\left(E^{n}\right)$ by the adjoint isomorphisms, and $\operatorname{GL}\left(n, \mathbb{Z}_{2}\right)$ acts canonically on $\mathbb{Z}_{2}\left[y_{1}, \ldots, y_{n}\right]$.

The invariant ring of $\operatorname{GL}\left(n, \mathbb{Z}_{2}\right)$ has been determined previously by Dickson [3] as follows. Let $Q_{k, 0}, \ldots, Q_{k, k-1}$ be the polynomials of $y_{1}, \ldots, y_{k}$ given by the inductive formula

$$
Q_{n, s}=\left(\eta_{n} Q_{n-1, s}\right) \cdot V_{n, n}+\eta_{n} Q_{n-1, s-1}^{2}, \quad 0 \leq s<n,
$$

where $V_{n, n}$ is defined in (2.14), $Q_{k, k}=1 \quad(k \geq 0)$ by convention, and $\eta_{n}$ is the following transformation of variable

$$
\eta_{n}=\left(\begin{array}{llll}
0 & & & 1 \\
& & . \cdot & \\
& 1 & & \\
1 & & & 0
\end{array}\right) \in \operatorname{GL}\left(n, \mathbb{Z}_{2}\right) .
$$

Then, $Q_{n, 0}, \ldots, Q_{n, n-1}$ are $\operatorname{GL}\left(n, \mathbb{Z}_{2}\right)$-invariants and Dickson has proved that

$$
\mathbb{Z}_{2}\left[y_{1}, \ldots, y_{n}\right]^{\mathrm{GL}\left(n, \mathbb{Z}_{2}\right)}=\mathbb{Z}_{2}\left[Q_{n, 0}, \ldots, Q_{n, n-1}\right] .
$$

Further, according to Quillen, Milgram, Huỳnh Mùi (see [18], [6; Appendix]) we have

$$
\operatorname{Res}\left(E^{n}, \mathfrak{S}_{2^{n}}\right) W_{n, s}=Q_{n, s}, \quad 0 \leq s<n .
$$

3.14. Lemma. Let $\left(W_{n, 0}\right)$ denote the ideal of $H^{*}\left(\mathfrak{S}_{2^{n}}\right)$ generated by $W_{n, 0}$. Then we have

$$
\operatorname{Res}\left(\mathfrak{S}_{2^{n}, 2}, \mathfrak{S}_{2^{n}}\right)\left(\left(W_{n, 0}\right)\right) \subset \mathbb{Z}_{2}\left[\bar{V}_{n, 1}, \ldots, \bar{V}_{n, n}\right] .
$$

Proof. Suppose that we get

$$
\begin{aligned}
& \bar{Q}_{n, 0} \cdot \mathscr{M}^{\perp}(\infty, n)^{*}=0, \\
& \bar{Q}_{n, 0}=\bar{V}_{n, 1} \cdots \bar{V}_{n, n} .
\end{aligned}
$$

Then, combining these with 2.8 and 2.10 , we prove the lemma as follows.

$$
\begin{gathered}
\operatorname{Res}\left(\mathfrak{S}_{2^{n}, 2}, \mathfrak{S}_{2^{n}}\right)\left(\left(W_{n, 0}\right)\right)=\operatorname{Res}\left(\mathfrak{S}_{2^{n}, 2}, \mathfrak{S}_{2^{n}}\right)\left(W_{n, 0} \cdot H^{*}\left(\mathfrak{S}_{2^{n}}\right)\right) \\
\subset \bar{Q}_{n, 0} \cdot H^{*}\left(\mathfrak{S}_{2^{n}, 2}\right)=\bar{Q}_{n, 0}\left(\mathscr{M}^{\perp}(\infty, n)^{*} \oplus \mathscr{M}(\infty, n)^{*}\right) \\
=\bar{Q}_{n, 0} \cdot \mathbb{Z}_{2}\left[\bar{V}_{n, 1}, \ldots, \bar{V}_{n, n}\right] \subset \mathbb{Z}_{2}\left[\bar{V}_{n, 1}, \ldots, \bar{V}_{n, n}\right] .
\end{gathered}
$$

Hence, it suffices to verify (3.15). To this end, following Proposition 3.8 we consider the restrictions of the elements in (3.15) to every maximal elementary abelian 2-subgroup of $\mathfrak{S}_{2^{n}, 2}$. 
According to Nakamura (see [6; II.2.7]) such a subgroup is conjugate in $\mathfrak{S}_{2^{n}, 2}$ to a certain group $A$ being of the following two kinds:

(a) First kind: $A$ is a maximal elementary abelian 2-subgroup of $\mathfrak{S}_{2^{n-1}, 2}^{2}$,

(b) Second kind: $A=A^{\prime} \times E_{n}$, where $A^{\prime}$ is a maximal elementary abelian 2-subgroup of the diagonal $\mathfrak{S}_{2^{n-1}, 2}$ in $\mathfrak{S}_{2^{n-1}, 2}^{2}$.

First we show that

$$
\operatorname{Res}\left(A, \mathfrak{S}_{2^{n}, 2}\right) Q_{n, 0}=0
$$

for every maximal elementary abelian 2-subgroup $A$ which is not conjugate to $E^{n}$ in $\mathfrak{S}_{2^{n}, 2}$.

By [6; II.2.8] and by a simple computation of rank of groups, we note that such a group $A$ is a subgroup of a certain maximal elementary abelian 2-subgroup $A_{1}$ of $\mathfrak{S}_{2^{n}}$ which is not conjugate to $E^{n}$ in $\mathfrak{S}_{2^{n}}$. By [6; II.2.3], $A_{1}$ is conjugate in $\mathfrak{S}_{2^{n}}$ to a subgroup $A_{2}$ of $\mathfrak{S}_{2^{n-1}}^{2}$. Since $\mathfrak{S}_{2^{n-1}, 2}$ is a Sylow 2-subgroup of $\mathfrak{S}_{2^{n-1}}$ so $A_{2}$ is conjugate in $\mathfrak{S}_{2^{n-1}}^{2}$ to a subgroup of $\mathfrak{S}_{2^{n-1}, 2}^{2}$. Thus, $A$ is conjugate in $\mathfrak{S}_{2^{n}}$ to a certain subgroup of $\mathfrak{S}_{2^{n-1}, 2}^{2}$.

In $[15 ; 3.4]$ we have proved that

$$
\operatorname{Res}\left(\mathfrak{S}_{2^{n-1}}^{2}, \mathfrak{S}_{2^{n}}\right) W_{n, 0}=0 .
$$

Since the diagram of restrictions

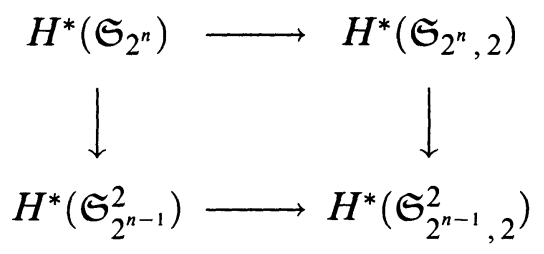

is commutative, we obtain

$$
\operatorname{Res}\left(\mathfrak{S}_{2^{n-1}, 2}^{2}, \mathfrak{S}_{2^{n}, 2}\right) \bar{Q}_{n, 0}=\operatorname{Res}\left(\mathfrak{S}_{2^{n-1}, 2}^{2}, \mathfrak{S}_{2^{n}}\right) W_{n, 0}=0
$$

By the above discussion and from the fact that

$$
\bar{Q}_{n, 0} \in \operatorname{Im} \operatorname{Res}\left(\mathfrak{S}_{2^{n}, 2}, \mathfrak{S}_{2^{n}}\right),
$$

it implies (3.16).

Combine (3.16) with

$$
\operatorname{Res}\left(E^{n}, \mathfrak{S}_{2^{n}, 2}\right) \mathscr{M}^{\perp}(\infty, n)^{*}=0
$$

as seen in 2.16 , we obtain the first equality of (3.15). 
Next we prove that

$$
\operatorname{Res}\left(A, \mathfrak{S}_{2^{n}, 2}\right) \bar{V}_{n, 1} \cdots \bar{V}_{n, n}=0,
$$

for $A$ as in 3.16.

From 2.2 and 2.3 we note that $\underbrace{0, \ldots, 0,1}_{n}=\bar{V}_{n, 1}^{*}$ does not belong to the image of the homomorphism

$$
H_{*}\left(\mathfrak{S}_{2^{n-1}, 2}^{2}\right) \rightarrow H_{*}\left(\mathfrak{S}_{2^{n}, 2}\right)
$$

induced by the inclusion $\mathfrak{S}_{2^{n-1}, 2}^{2} \subset \mathfrak{S}_{2^{n}, 2}$. So we have

$$
\operatorname{Res}\left(\mathfrak{S}_{2^{n-1}, 2}^{2}, \mathfrak{S}_{2^{n}, 2}\right) \bar{V}_{n, 1}=0 .
$$

This implies (3.17) in the case where $A$ is of the first kind.

Let $A=A^{\prime} \times E_{n}$ be of the second kind and not conjugate to $E^{n}$ in $\mathfrak{S}_{2^{n}, 2}$. So $A^{\prime}$ is not conjugate to $E^{n-1}$ in $\mathfrak{S}_{2^{n-1}, 2}$. We prove (3.17) by induction on $n$. For $n=1$, the problem is trivial because there exists no such subgroup $A$ in $\mathfrak{S}_{2,2}=E$. Suppose $n>1$ and (3.17) is true for $n-1$. Let us regard the commutative diagram

$$
\begin{array}{cccc}
H^{*}\left(\mathfrak{S}_{2^{n-1}, 2}\right) & \stackrel{P}{\longrightarrow} H^{*}\left(\mathfrak{S}_{2^{n}, 2}\right) & \stackrel{d^{*}=\text { Res }}{\longrightarrow} H^{*}\left(\mathfrak{S}_{2^{n-1}, 2} \times E_{n}\right) \\
\downarrow & \downarrow \text { Res } & & \downarrow \text { Res } \\
H^{*}\left(A^{\prime}\right) & \stackrel{P}{\longrightarrow} H^{*}\left(A^{\prime} \int E_{n}\right) \stackrel{d^{*}=\text { Res }}{\longrightarrow} & H^{*}\left(A^{\prime} \times E_{n}\right),
\end{array}
$$

where $P$ 's denote the Steenrod maps. As seen in (2.12), we have

$$
\bar{V}_{n, r}=P\left(\bar{V}_{n-1, r-1}\right), \quad 1<r \leq n .
$$

Using the diagram and the inductive hypothesis, we obtain

$$
\begin{aligned}
& \operatorname{Res}\left(A, \mathfrak{S}_{2^{n}, 2}\right) \bar{V}_{n, 1} \cdots \bar{V}_{n, n} \\
& \quad=\operatorname{Res}\left(A, \mathfrak{S}_{2^{n}, 2}\right)\left(\bar{V}_{n, 1} \cdot P\left(\bar{V}_{n-1,1} \cdots \bar{V}_{n-1, n-1}\right)\right) \\
& =\operatorname{Res}\left(A, \mathfrak{S}_{2^{n}, 2}\right)\left(\bar{V}_{n, 1}\right) d^{*} P \operatorname{Res}\left(A^{\prime}, \mathfrak{S}_{2^{n-1}, 2}\right)\left(\bar{V}_{n-1,1} \cdots \bar{V}_{n-1, n-1}\right) \\
& =0 .
\end{aligned}
$$

This completes the proof of (3.17).

At last, we prove

$$
\operatorname{Res}\left(E^{n}, \mathfrak{S}_{2^{n}, 2}\right) \bar{Q}_{n, 0}=\operatorname{Res}\left(E^{n}, \mathfrak{S}_{2^{n}, 2}\right) \bar{V}_{n, 1} \cdots \bar{V}_{n, n} .
$$

In the other words, we must show that

$$
Q_{n, 0}=V_{n, 1} \cdots V_{n, n} \text {. }
$$


We set $V_{r}=\eta_{n} V_{n, r}$ for $1 \leq r \leq n$. From (2.14) we note that $V_{r}$ depends only on $y_{1}, \ldots, y_{r}$. By means of (3.10) we get

$$
Q_{n, 0}=Q_{n-1,0} V_{n}=V_{1} \cdots V_{n} \text {. }
$$

This implies

$$
Q_{n, 0}=\eta_{n} Q_{n, 0}=V_{n, 1} \cdots V_{n, n} .
$$

Combining (3.16), (3.17) and (3.18) we have the second equality of (3.15). The proof of the lemma is completed.

Proof of Proposition 3.5. According to [15; 3.4 and 3.8] we get

$$
\operatorname{Ker} \operatorname{Res}\left(\mathfrak{S}_{2^{n-1}}^{2}, \mathfrak{S}_{2^{n}}\right)=\left(W_{n, 0}\right)=W_{n, 0} \mathbb{Z}_{2}\left[W_{n, 0}, \ldots, W_{n, n-1}\right] .
$$

It implies from (3.14) that

$$
\begin{aligned}
i^{*}(\infty, n)\left(\left(W_{n, 0}\right)\right) & =\bar{Q}_{n, 0} \mathbb{Z}_{2}\left[\bar{Q}_{n, 0}, \ldots, \bar{Q}_{n, n-1}\right] \\
& \subset \mathbb{Z}_{2}\left[\bar{V}_{n, 1}, \ldots, \bar{V}_{n, n}\right] .
\end{aligned}
$$

On the other hand, by Theorem 2.10 , we obtain

$$
\begin{aligned}
& i^{*}(M, q) \mathbb{Z}_{2}\left[\bar{V}_{n, 1}, \ldots, \bar{V}_{n, n}\right] \\
& \quad=\mathbb{Z}_{2}\left[\bar{V}_{n, 1}, \ldots, \bar{V}_{n, n}\right] /\left(\bar{V}_{n, 1}^{q}, \ldots, \bar{V}_{n, n}^{q}\right) .
\end{aligned}
$$

By Proposition 2.16, the homomorphism

$$
\left.d_{n}^{*}\right|_{\mathscr{M}(\infty, n)^{*}}: \mathbb{Z}_{2}\left[\bar{V}_{n, 1}, \ldots, \bar{V}_{n, n}\right] \rightarrow \mathbb{Z}_{2}\left[V_{n, 1}, \ldots, V_{n, n}\right]
$$

is an isomorphism. Further, we have

$$
d_{n}^{*}\left(\bar{V}_{n, r}\right)=V_{n, r} \quad(1 \leq r \leq n), \quad d_{n}^{*}\left(\bar{Q}_{n, s}\right)=Q_{n, s} \quad(0 \leq s<n) .
$$

From the above discussion, Proposition 3.5 is proved by the following.

\subsection{Lemma. Let}

$$
\text { pr: } \mathbb{Z}_{2}\left[V_{n, 1}, \ldots, V_{n, n}\right] \rightarrow \mathbb{Z}_{2}\left[V_{n, 1}, \ldots, V_{n, n}\right] /\left(V_{n, 1}^{q}, \ldots, V_{n, n}^{q}\right)
$$

be the projection. Then, for the subring $\mathbb{Z}_{2}\left[Q_{n, 0}, \ldots, Q_{n, n-1}\right]$ of $\mathbb{Z}_{2}\left[V_{n, 1}, \ldots, V_{n, n}\right]$, we have

$$
\operatorname{pr} \mathbb{Z}_{2}\left[Q_{n, 0}, \ldots, Q_{n, n-1}\right]=\mathbb{Z}_{2}\left[Q_{n, 0}, \ldots, Q_{n, n-1}\right] / I(Q, q) .
$$

Here $I(Q, q)$ denotes the ideal of $\mathbb{Z}_{2}\left[Q_{n, 0}, \ldots, Q_{n, n-1}\right]$ generated by monomials of degree $q$.

Proof. Let $\eta_{n}: \mathbb{Z}_{2}\left[y_{1}, \ldots, y_{n}\right] \stackrel{\simeq}{\rightarrow} \mathbb{Z}_{2}\left[y_{1}, \ldots, y_{n}\right]$ be the ring isomorphism induced by the transformation of variable $\eta_{n}$ given in 
(3.11). Since $Q_{n, s}$ is an invariant of $\operatorname{GL}\left(n, \mathbb{Z}_{2}\right)$, we observe that $\eta_{n} Q_{n, s}=Q_{n, s}$ for $0 \leq s<n$. We set

$$
V_{r}=\eta_{n} V_{n, r}, \quad 1 \leq r \leq n .
$$

Then, the lemma admits the equivalent form as follows. Let

$$
\text { pr: } \mathbb{Z}_{2}\left[V_{1}, \ldots, V_{n}\right] \rightarrow \mathbb{Z}_{2}\left[V_{1}, \ldots, V_{n}\right] /\left(V_{1}^{q}, \ldots, V_{n}^{q}\right)
$$

be the projection. We must prove that

$$
\operatorname{pr} \mathbb{Z}_{2}\left[Q_{n, 0}, \ldots, Q_{n, n-1}\right]=\mathbb{Z}_{2}\left[Q_{n, 0}, \ldots, Q_{n, n-1}\right] / I(Q, q) .
$$

The proof proceeds by induction on $n$. The lemma is trivial for $n=1$. Suppose that $n>1$ and it is true for $n-1$. Note that $Q_{n-1,0}, \ldots, Q_{n-1, n-2}$ are algebraically independent and they depend only on $y_{1}, \ldots, y_{n-1}$. Meanwhile, $V_{n}$ depends not only on $y_{1}, \ldots$, $y_{n-1}$ but also $y_{n}$. Hence, the system $Q_{n-1,0}, \ldots, Q_{n-1, n-2}, V_{n}$ is algebraically independent.

From the Dickson formula (3.10) and the fact that $\eta_{n}^{2}=1$, we get

$$
Q_{n, s}=Q_{n-1, s} \cdot V_{n}+Q_{n-1, s-1}^{2} \text {. }
$$

It implies that

$$
\begin{aligned}
\mathbb{Z}_{2}\left[Q_{n, 0}, \ldots, Q_{n, n-1}\right] & \subset \mathbb{Z}_{2}\left[Q_{n-1,0}, \ldots, Q_{n-1, n-2}, V_{n}\right] \\
& \subset \mathbb{Z}_{2}\left[V_{1}, \ldots, V_{n}\right] .
\end{aligned}
$$

Further, we have the commutative diagram

$$
\begin{gathered}
\mathbb{Z}_{2}\left[V_{1}, \ldots, V_{n-1}\right] \stackrel{\text { pr }}{\longrightarrow} \mathbb{Z}_{2}\left[V_{1}, \ldots, V_{n-1}\right] /\left(V_{1}^{q}, \ldots, V_{n-1}^{q}\right) \\
\downarrow \\
\mathbb{Z}_{2}\left[V_{1}, \ldots, V_{n}\right] \stackrel{\text { pr }}{\longrightarrow} \mathbb{Z}_{2}\left[V_{1}, \ldots, V_{n}\right] /\left(V_{1}^{q}, \ldots, V_{n}^{q}\right),
\end{gathered}
$$

where the vertical arrows are the canonical injections. From this diagram and the inductive hypothesis, we obtain

$$
\operatorname{pr} \mathbb{Z}_{2}\left[Q_{n-1,0}, \ldots, Q_{n-1, n-s}, V_{n}\right]=\mathbb{Z}_{2}\left[Q_{n-1,0}, \ldots, Q_{n-1, n-2}, V_{n}\right] / I
$$

where $I$ denotes the ideal of $\mathbb{Z}_{2}\left[Q_{n-1,0}, \ldots, Q_{n-1, n-2}, V_{n}\right]$ generated by $V_{n}^{q}$ and monomials of $Q_{n-1,0}, \ldots, Q_{n-1, n-2}$ of degree $q$. At last, using the Dickson formula (3.20), we can verify directly that

$$
\mathbb{Z}_{2}\left[Q_{n, 0}, \ldots, Q_{n, n-1}\right] \cap I=I(Q, q) .
$$

This completes the proof of Lemma 3.19. Proposition 3.5 follows. 
4. The algebras $H^{*}\left(F\left(\mathbb{R}^{q}, m\right) / \mathfrak{S}_{m}\right)$. In this section, we shall determine the algebras $H^{*}\left(F\left(\mathbb{R}^{q}, m\right) / \mathfrak{S}_{m}\right)$ by means of the universal Dickson characteristic classes introduced in [15].

Remember that, according to Huỳnh Mùi's result on $\operatorname{Res}\left(E^{n}, \mathfrak{S}_{2^{n}}\right)$ (see (3.9), (3.12), (3.13)), we get

$$
H^{*}\left(\mathfrak{S}_{2^{n}}\right)=\operatorname{Ker} \operatorname{Res}\left(E^{n}, \mathfrak{S}_{2^{n}}\right) \oplus \mathbb{Z}_{2}\left[W_{n, 0}, \ldots, W_{n, n-1}\right] .
$$

We defined in $[13 ; 2.4]$ and $[15 ; 3.5]$ the Dickson elements $D_{k_{0}, \ldots, k_{n-1}}$ in $H_{*}\left(\mathfrak{S}_{2^{n}}\right) \subset H_{*}\left(\mathfrak{S}_{\infty}\right)$, for $k_{i} \geq 0$, by the conditions

$$
\begin{aligned}
& \left\langle D_{k_{0}, \ldots, k_{n-1}}, \operatorname{Ker} \operatorname{Res}\left(E^{n}, \mathfrak{S}_{2^{n}}\right)\right\rangle=0, \\
& \left\langle D_{k_{0}, \ldots, k_{n-1}}, \prod_{s=0}^{n-1} W_{n, s}^{h_{s}}\right\rangle= \begin{cases}1, & \left(k_{0}, \ldots, k_{n-1}\right)=\left(h_{0}, \ldots, h_{n-1}\right), \\
0, & \text { otherwise. }\end{cases}
\end{aligned}
$$

Here and from now on, $\langle\cdot, \cdot\rangle$ denotes the dual pairing. Then we obtained

4.2 Theorem [13;2.5], [15; 3.7 and 3.8].

(i) $H_{*}\left(\mathfrak{S}_{\infty}\right)=\mathbb{Z}_{2}\left[D_{K} ; K \in J^{+}(\infty)\right]$ as algebras with multiplicity. Here the multiplicity of $D_{k_{0}}, \ldots, k_{n-1}$ is given to be $2^{n}$. So we have an isomorphism of $\mathbb{Z}_{2}$-modules for arbitrary $m$

$$
H_{*}\left(\mathfrak{S}_{m}\right) \cong \mathbb{Z}_{2}\left[D_{K} ; K \in J^{+}(\infty)\right](m) .
$$

The basis of this module consisting of all monomials in

$$
\mathbb{Z}_{2}\left[D_{K} ; K \in J^{+}(\infty)\right]
$$

of multiplicities $\leq m$ will be called the Dickson basis.

(ii) The comultiplication $\Delta$ of the Hopf algebra $H_{*}\left(\mathfrak{S}_{\infty}\right)$ satisfies the formula

$$
\Delta D_{k_{0}, \ldots, k_{n-1}}=\sum_{l_{i}+m_{t}=k_{l}} D_{l_{0}, \ldots, l_{n-1}} \otimes D_{m_{0}, \ldots, m_{n-1}}
$$

for $k_{i}, l_{i}, m_{i} \geq 0,0 \leq i<n$.

(iii) We have the reduction formula

$$
D^{0, \ldots, 0, k_{0}, \ldots, k_{n-1}}=D_{k_{0}, \ldots, k_{n-1}}^{2^{s}} .
$$

In $[15 ; 3.4]$ we have shown that, under the identification $H^{*}\left(\mathfrak{S}_{2^{n}}\right)=$ $H^{*}\left(F\left(\mathbb{R}^{\infty}, 2^{n}\right) / \mathfrak{S}_{2^{n}}\right), W_{n, s}$ coincides with the $\left(2^{n}-2^{s}\right)$-the StiefelWhitney class of the vector bundle

$$
\text { pr: } \mathbb{R}^{2^{n}} \times_{\mathfrak{S}_{2^{n}}} F\left(\mathbb{R}^{\infty}, 2^{n}\right) \rightarrow F\left(\mathbb{R}^{\infty}, 2^{n}\right) / \mathfrak{S}_{2^{n}}
$$


for $0 \leq s<n$. Here the group $\mathfrak{S}_{2^{n}}$ acts on $\mathbb{R}^{2^{n}}$ by permutations of coordinates. So we shall denote also by $W_{n, s}$, for $0 \leq s<n$, the $\left(2^{n}-2^{s}\right)$ th Stiefel-Whitney class of the vector bundle

$$
\text { pr: } \mathbb{R}^{2^{n}} \times_{\mathfrak{S}_{2^{n}}} F\left(\mathbb{R}^{q}, 2^{n}\right) \rightarrow F\left(\mathbb{R}^{q}, 2^{n}\right) / \mathfrak{S}_{2^{n}}
$$

Obviously we have

$$
\begin{aligned}
i^{*}(F, q): H^{*}\left(F\left(\mathbb{R}^{\infty}, 2^{n}\right) / \mathfrak{S}_{2^{n}}\right) & \rightarrow H^{*}\left(F\left(\mathbb{R}^{q}, 2^{n}\right) / \mathfrak{S}_{2^{n}}\right), \\
W_{n, s} & \mapsto W_{n, s}, \quad 0 \leq s<n .
\end{aligned}
$$

To describe the structure of the Hopf algebra $H_{*}\left(F\left(\mathbb{R}^{q}, \infty\right) / \mathfrak{S}_{\infty}\right)$ we need the following two lemmas, which will be proved by use of the embeddings $i(q, n): M(q, n) \rightarrow F\left(\mathbb{R}^{q}, 2^{n}\right) / \mathfrak{S}_{2^{n}}$ as a main tool.

\subsection{LEMMA.}

$$
i^{*}(F, q)\left(\left(W_{n, 0}\right)\right)=W_{n, 0} \mathbb{Z}_{2}\left[W_{n, 0}, \ldots, W_{n, n-1}\right] / I(W, q),
$$

where $I(W, q)$ denotes the ideal of $W_{n, 0} \mathbb{Z}_{2}\left[W_{n, 0}, \ldots, W_{n, n-1}\right]$ generated by monomials of degree $q$.

Proof. From Diagram (3.7) and Proposition 3.5 it implies

$$
\begin{aligned}
i^{*}(q, & n) i^{*}(F, q)\left(\left(W_{n, 0}\right)\right)=i^{*}(M, q) i^{*}(\infty, n)\left(\left(W_{n, 0}\right)\right) \\
& =i^{*}(M, q) i^{*}(\infty, n) \operatorname{Ker} \operatorname{Res}\left(\mathfrak{S}_{2^{n-1}}^{2}, \mathfrak{S}_{2^{n}}\right) \quad(\text { see }[15 ; 3.4 \text { and 3.8] }), \\
& =\bar{Q}_{n, 0} \mathbb{Z}_{2}\left[\bar{Q}_{n, 0}, \ldots, \bar{Q}_{n, n-1}\right] / I(\bar{Q}, q) .
\end{aligned}
$$

According to $3.1, i^{*}(q, n)$ is a monomorphism. So we get

$$
i^{*}(F, q)\left(\left(W_{n, 0}\right)\right)=W_{n, 0} \mathbb{Z}_{2}\left[W_{n, 0}, \ldots, W_{n, n-1}\right] / I(W, q) .
$$

The lemma is proved.

4.5. Lemma. The image of the monomorphism

$$
i_{*}(F, q): H_{*}\left(F\left(\mathbb{R}^{q}, \infty\right) / \mathfrak{S}_{\infty}\right) \rightarrow H_{*}\left(F\left(\mathbb{R}^{\infty}, \infty\right) / \mathfrak{S}_{\infty}\right) .
$$

is given by

$$
\operatorname{Im} i_{*}(F, q)=\mathbb{Z}_{2}\left[D_{K} ; K \in J^{+}(q)\right], \quad 1 \leq q \leq \infty .
$$

Via this monomorphism, $H_{*}\left(F\left(\mathbb{R}^{q}, \infty\right) / \mathfrak{S}_{\infty}\right)$ becomes a Hopf subalgebra as well as a subalgebra with multiplicity of $H_{*}\left(F\left(\mathbb{R}^{\infty}, \infty\right) / \mathfrak{S}_{\infty}\right)$. 
Hence as a consequence of 4.2 and 4.5 we have

4.6. THEOREM. (i) $H_{*}\left(F\left(\mathbb{R}^{q}, \infty\right) / \mathfrak{S}_{\infty}\right)=\mathbb{Z}_{2}\left[D_{K} ; K \in J^{+}(q)\right]$ as algebras with multiplicity for $q>0$. Here $D_{k_{0}, \ldots, k_{n-1}}$ is of multiplicity $2^{n}$. So we have the isomorphism of $\mathbb{Z}_{2}$-modules for every $m$

$$
H_{*}\left(F\left(\mathbb{R}^{q}, m\right) / \mathfrak{S}_{m}\right)=\mathbb{Z}_{2}\left[D_{K} ; K \in J^{+}(q)\right](m) .
$$

The Dickson basis for this module is defined similarly as in 4.2.

(ii) Let

$$
J(q)=\left\{\left(k_{0}, \ldots, k_{n-1}\right) \neq 0 ; n>0, k_{i} \in \mathbb{Z}_{+}, k_{0}+\cdots+k_{n-1}<q\right\} .
$$

The comultiplication $\Delta$ of the Hopf algebra $H_{*}\left(F\left(\mathbb{R}^{q}, \infty\right) / \mathfrak{S}_{\infty}\right)$ is given by

$$
\Delta D_{k_{0}, \ldots, k_{n-1}}=\sum_{l_{\imath}+m_{\imath}=k_{t}} D_{l_{0}, \ldots, l_{n-1}} \otimes D_{m_{0}, \ldots, m_{n-1}}
$$

for $\left(k_{0}, \ldots, k_{n-1}\right) \in J(q), l_{i}, m_{i} \geq 0,0 \leq i<n$.

(iii)

$$
D_{\underbrace{0, \ldots, 0,}_{s}}^{0, \ldots, k_{n-1}}=D_{k_{0}, \ldots, k_{n-1}}^{2^{s}}
$$

for $s \geq 0,\left(k_{0}, \ldots, k_{n-1}\right) \in J(q)$.

Proof of Lemma 4.5. Set

$$
R=\operatorname{Ker} \operatorname{Res}\left(E^{n}, \mathfrak{S}_{2^{n}}\right) \oplus \mathbb{Z}_{2}\left[W_{n, 1}, \ldots, W_{n, n-1}\right] .
$$

We obtain clearly $H^{*}\left(\mathfrak{S}_{2^{n}}\right)=R \oplus\left(W_{n, 0}\right)$.

First, we are going to show

$$
H^{*}\left(F\left(\mathbb{R}^{q}, 2^{n}\right) / \mathfrak{S}_{2^{n}}\right)=i^{*}(F, q) R \oplus i^{*}(F, q)\left(\left(W_{n, 0}\right)\right) .
$$

By Theorem 3.4, $i^{*}(F, q)$ is an epimorphism. So it suffices to prove

$$
i^{*}(F, q) R \cap i^{*}(F, q)\left(\left(W_{n, 0}\right)\right)=\{0\} .
$$

Since $i^{*}(q, n)$ is a monomorphism, this is equivalent to

$$
i^{*}(q, n) i^{*}(F, q) R \cap i^{*}(q, n) i^{*}(F, q)\left(\left(W_{n, 0}\right)\right)=\{0\},
$$

or equivalent to (by Diagram (3.7))

$$
i^{*}(M, q) i^{*}(\infty, n) R \cap i^{*}(M, q) i^{*}(\infty, n)\left(\left(W_{n, 0}\right)\right)=\{0\} .
$$

From Propositions 3.5 and 2.16 it implies

$$
i^{*}(M, q) i^{*}(\infty, n)\left(\left(W_{n, 0}\right)\right)=\bar{Q}_{n, 0} \mathbb{Z}_{2}\left[\bar{Q}_{n, 0}, \ldots, \bar{Q}_{n, n-1}\right] / I(\bar{Q}, q),
$$

$$
\begin{aligned}
& i^{*}(M, q) i^{*}(\infty, n) \operatorname{Ker} \operatorname{Res}\left(E^{n}, \mathfrak{S}_{2^{n}}\right) \\
& \quad \subset i^{*}(M, q) \mathscr{M}^{\perp}(\infty, n)^{*} \subset \mathscr{M}^{\perp}(q, n)^{*} .
\end{aligned}
$$


Let us consider the diagram

$$
\begin{aligned}
& H^{*}(M(\infty, n)) \longleftarrow \mathbb{Z}_{2}\left[V_{n, 1}, \ldots, V_{n, n}\right] \\
& \downarrow i^{*}(M, q) \quad \downarrow \text { pr } \\
& H^{*}(M(q, n)) \stackrel{\alpha^{\prime}}{\longleftarrow} \mathbb{Z}_{2}\left[V_{n, 1}, \ldots, V_{n, n}\right] /\left(V_{n, 1}^{q}, \ldots, V_{n, n}^{q}\right),
\end{aligned}
$$

where $\alpha$ denotes the split given in 2.15 and $\alpha^{\prime}$ is defined so that the diagram is commutative (see 2.10). We have

$$
\begin{aligned}
\alpha\left(Q_{n, s}\right) & =\bar{Q}_{n, s} \bmod \mathscr{M}^{\perp}(\infty, n)^{*}, \\
\alpha^{\prime}\left(\operatorname{pr} Q_{n, s}\right) & =i^{*}(M, q) \bar{Q}_{n, s} \bmod \mathscr{M}^{\perp}(q, n)^{*},
\end{aligned}
$$

for $0 \leq s<n$. From this and Lemma 3.19 we obtain

$$
\begin{aligned}
& i^{*}(M, q) i^{*}(\infty, n) \mathbb{Z}_{2}\left[W_{n, 1}, \ldots, W_{n, n-1}\right] \\
& \quad=i^{*}(M, q) \mathbb{Z}_{2}\left[\bar{Q}_{n, 1}, \ldots, \bar{Q}_{n, n-1}\right] \\
& \quad=\mathbb{Z}_{2}\left[\bar{Q}_{n, 1}, \ldots, \bar{Q}_{n, n-1}\right] / J \bmod \mathscr{M}^{\perp}(q, n)^{*},
\end{aligned}
$$

where $J$ is the ideal of $\mathbb{Z}_{2}\left[\bar{Q}_{n, 1}, \ldots, \bar{Q}_{n, n-1}\right]$ generated by monomials of degree $q$.

Combining (4.8) and (4.9) we get (4.7).

Now for $K=\left(k_{0}, \ldots, k_{n-1}\right) \in J^{+}(q)$ we can define the element (which is also denoted by) $D_{K}=D_{k_{0}, \ldots, k_{n-1}}$ in $H_{*}\left(F\left(\mathbb{R}^{q}, 2^{n}\right) / \mathfrak{S}_{2^{n}}\right) \subset$ $H_{*}\left(F\left(\mathbb{R}^{q}, \infty\right) / \mathfrak{S}_{\infty}\right)$ by the conditions

$$
\left\langle D_{k_{0}, \ldots, k_{n-1}}, i^{*}(F, q) R\right\rangle=0,
$$

$$
\left\langle D_{k_{0}, \ldots, k_{n-1}}, \prod_{s=0}^{n-1} W_{n, s}^{h_{s}}\right\rangle= \begin{cases}1, & \left(k_{0}, \ldots, k_{n-1}\right)=\left(h_{0}, \ldots, h_{n-1}\right), \\ 0 & \text { otherwise. }\end{cases}
$$

From (4.1), (4.3) and (4.10) it is easy to see that

$$
\begin{aligned}
i_{*}(F, q): H_{*}\left(F\left(\mathbb{R}^{q}, \infty\right) / \mathfrak{S}_{\infty}\right) & \rightarrow H_{*}\left(F\left(\mathbb{R}^{\infty}, \infty\right) / \mathfrak{S}_{\infty}\right), \\
D_{K} & \mapsto D_{K},
\end{aligned}
$$

for $K \in J^{+}(q)$.

Combining this with Theorem 4.2 it implies that

$$
\operatorname{Im} i_{*}(F, q) \supset \mathbb{Z}_{2}\left[D_{K} ; K \in J^{+}(q)\right],
$$

since the image is a subalgebra of $H_{*}\left(F\left(\mathbb{R}^{\infty}, \infty\right) / \mathfrak{S}_{\infty}\right)$. Moreover, by Theorem 3.4, both domains of this inclusion are isomorphic to each other as graded $\mathbb{Z}_{2}$-modules of finite type via the formal corresponding

$$
N_{K} \leftrightarrow D_{K}, \quad K \in J^{+}(q) .
$$


So they are coincident. The proof of Lemma 4.5 is completed.

Recall that in $[13 ; \S 3]$ and $[15 ; \S 4]$ we have introduced the universal Dickson class $W^{H}$ in $H^{*}\left(\mathfrak{S}_{\infty}\right)$, for $H \in J(\infty)$, which is dual to $D_{H}$ via the Dickson basis of $H_{*}\left(\mathfrak{S}_{\infty}\right)$. We recall that

$$
\operatorname{Res}\left(\mathfrak{S}_{2^{n}}, \mathfrak{S}_{\infty}\right) W^{H}=\prod_{s=0}^{n-1} W_{n, s}^{h_{s}}
$$

if $H=\left(h_{0}, \ldots, h_{n-1}\right)$. Further, we have proved in $[15 ; 4.10]$

$$
H^{*}\left(\mathfrak{S}_{\infty}\right)=\mathbb{Z}_{2}\left[W^{H} ; H \in J_{\text {odd }}(\infty)\right]
$$

as algebras, with $J_{\text {odd }}(\infty)$ as mentioned in the introduction.

Note that from Lemma 4.5 it implies

$$
i^{*}(F, q) W^{H} \neq 0 \Leftrightarrow H \in J(q) .
$$

For simplicity, the Dickson class $i^{*}(F, q) W^{H}$ of the map $i(F, q)$ will be denoted also by $W^{H}$, for $H \in J(q)$. This is dual to $D_{H}$ via the Dickson basis of $H_{*}\left(F\left(\mathbb{R}^{q}, \infty\right) / \mathfrak{S}_{\infty}\right)$, according to Lemma 4.5. Further, by (4.3) and (4.11), we have

$$
\left.W^{H}\right|_{F\left(\mathbb{R}^{q}, 2^{n}\right) / \mathfrak{S}_{2^{n}}}=\prod_{s=0}^{n-1} W_{n, s}^{h_{s}},
$$

for $H=\left(h_{0}, \ldots, h_{n-1}\right) \in J(q)$. We are now ready to get the main result of the paper.

4.15. THEOREM. We have an isomorphism of algebras

$$
\begin{aligned}
& H^{*}\left(F\left(\mathbb{R}^{q}, \infty\right) / \mathfrak{S}_{\infty}\right) \\
& \quad \cong \mathbb{Z}_{2}\left[W^{H} ; H \in J_{\text {odd }}(q)\right] /\left(\left(W^{H}\right)^{2^{h(q, H)}} ; H \in J_{\text {odd }}(q)\right)
\end{aligned}
$$

for $1 \leq q \leq \infty$. Here $J_{\text {odd }}(q)$ is as given in the introduction, and

$$
\begin{array}{r}
h(q, H)=\min \left\{h \in \mathbb{N}: 2^{h}\left(h_{0}+\cdots+h_{n-1}\right) \geq q\right\} \\
\qquad \text { for } H=\left(h_{0}, \ldots, h_{n-1}\right)
\end{array}
$$

Proof. According to Theorem 3.4, the restriction

$$
i^{*}(F, q): H^{*}\left(\mathfrak{S}_{\infty}\right)=H^{*}\left(F\left(\mathbb{R}^{\infty}, \infty\right) / \mathfrak{S}_{\infty}\right) \rightarrow H^{*}\left(F\left(\mathbb{R}^{q}, \infty\right) / \mathfrak{S}_{\infty}\right)
$$

is an epimorphism. By (4.12) and (4.13), this induces the surjection

$$
\mathbb{Z}_{2}\left[W^{H} ; H \in J_{\text {odd }}(q)\right] \rightarrow H^{*}\left(F\left(\mathbb{R}^{q}, \infty\right) / \mathfrak{S}_{\infty}\right) .
$$

In $[15 ; 4.9]$ by passing Theorem 4.2 to the dual we obtained in $H^{*}\left(\mathfrak{S}_{\infty}\right)$

$$
\left(W^{H}\right)^{2^{k}}=W^{2^{k} \cdot H} \quad \text { for } H \in J(\infty), \quad k \geq 0,
$$


where $2^{k} \cdot H=H+\cdots+H \quad\left(2^{k}\right.$ terms $)$. Here we must recall that, in [15; $\S 4$ ] we equipped $J(\infty)$ with a partial addition by agreeing that the sum $H+K$, where $H=\left(h_{0}, \ldots, h_{n-1}\right), K=\left(k_{0}, \ldots, h_{m-1}\right)$, is defined iff $n=m$; and in this case $H+K=\left(h_{0}+k_{0}, \ldots, h_{n-1}+k_{n-1}\right)$. Since $2^{h(q, H)} \cdot H$ is not in $J(q)$, from (4.13), (4.16) we have

$$
i^{*}(F, q)\left(W^{H}\right)^{2^{h(q, H)}}=0 .
$$

So we get the epimorphism induced by $i^{*}(F, q)$

$$
\begin{aligned}
& \mathbb{Z}_{2}\left[W^{H} ; H \in J_{\text {odd }}(q)\right] /\left(\left(W^{H}\right)^{2^{h(q, H)}} ; H \in J_{\text {odd }}(q)\right) \\
& \rightarrow H^{*}\left(F\left(\mathbb{R}^{q}, \infty\right) / \mathfrak{S}_{\infty}\right) .
\end{aligned}
$$

To prove that this is an isomorphism, it remains to show that both sides are isomorphic as graded modules (of finite type) over $\mathbb{Z}_{2}$.

There are isomorphisms of graded modules

$$
\begin{aligned}
H^{*}\left(F\left(\mathbb{R}^{q}, \infty\right) / \mathfrak{S}_{\infty}\right) & \cong H_{*}\left(F\left(\mathbb{R}^{q}, \infty\right) / \mathfrak{S}_{\infty}\right)=\mathbb{Z}_{2}\left[D_{K} ; K \in J^{+}(q)\right] \\
& \cong \mathbb{Z}_{2}\left[D_{2^{h} \cdot K} ; K \in J_{\text {odd }}^{+}(q), h<h(q, K)\right],
\end{aligned}
$$

where $J_{\text {odd }}^{+}(q)=J^{+}(q) \cap J_{\text {odd }}(q)$.

Note that we have

$$
\mathbb{Z}_{2}[x] \cong \bigotimes_{n \geq 0} \mathbb{Z}_{2}\left[x^{d^{n}}\right] /\left(x^{d^{n+1}}\right), \quad \text { for } d \in \mathbb{N}
$$

as graded modules. Applying this with $x=D_{2^{h} \cdot K}, d=2^{h(q, K)}$ we obtain an isomorphism of graded modules

$$
\begin{aligned}
& H^{*}\left(F\left(\mathbb{R}^{q}, \infty\right) / \mathfrak{S}_{\infty}\right) \\
& \cong \bigotimes_{n \geq 0} \frac{\mathbb{Z}_{2}\left[\left(D_{2^{h} \cdot K}\right)^{2^{h(q, K) n}} ; K \in J_{\text {odd }}^{+}(q), h<h(q, K)\right]}{\left(\left(D_{2^{h} \cdot K}\right)^{2^{h(q, K)(n+1)}} ; K \in J_{\text {odd }}^{+}(q), h<h(q, K)\right)} .
\end{aligned}
$$

Hinted by 4.6(iii) we define formally

$$
K^{2^{s}}=(\underbrace{0, \ldots, 0}_{0}, k_{0}, \ldots, k_{n-1}) \text { for } K=\left(k_{0}, \ldots, k_{n-1}\right) .
$$

As is easily seen, each $H \in J_{\text {odd }}(q)$ can be written uniquely in the form

$$
H=K^{2^{h+h(q, K) n}}
$$

for $K \in J_{\text {odd }}^{+}(q), h<h(q, K), n \geq 0$. This implies $h(q, H)=$ $h(q, K)$. So we have an isomorphism of graded modules

$$
\begin{aligned}
& H^{*}\left(F\left(\mathbb{R}^{q}, \infty\right) / \mathfrak{S}_{\infty}\right) \\
& \quad \cong \mathbb{Z}_{2}\left[W^{H} ; H \in J_{\text {odd }}(q)\right] /\left(\left(W^{H}\right)^{2^{h(q, H)}} ; H \in J_{\text {odd }}(q)\right),
\end{aligned}
$$


The theorem is proved.

$$
D_{2^{h} \cdot K}^{2^{h(q, K) n}} \mapsto W^{H} \quad \text { where } H=K^{2^{h+h(q, K) n}} .
$$

Now we prepare for determination of the algebras

$$
H^{*}\left(F\left(\mathbb{R}^{q}, m\right) / \mathfrak{S}_{m}\right) .
$$

For each $(\mathscr{H}, T)=\left(H_{1}, \ldots, H_{r}\right) \times\left(t_{1}, \ldots, t_{r}\right) \in J(q)^{r} \times \mathbb{N}^{r}$ we set

$$
W_{T}^{\mathscr{H}}=W_{t_{1}, \ldots, t_{r}}^{H_{1}, \ldots, H_{r}}=\left(D_{H_{1}}^{t_{1}} \cdots D_{H_{r}}^{t_{r}}\right)^{*} \in H^{*}\left(F\left(\mathbb{R}^{q}, \infty\right) / \mathfrak{S}_{\infty}\right),
$$

where the dual is defined by the Dickson basis. If $T=(1, \ldots, 1)$ ( $r$ times) we write simply $W^{\mathscr{H}}=W^{H_{1}}, \ldots, H_{r}$ instead of $W_{T}^{\mathscr{H}}$. This is compatible with the notation $W^{H}$ above.

For $\sigma \in \mathfrak{S}_{r}$ we have

$$
W_{T}^{\mathscr{P}}=W_{\sigma T}^{\sigma \mathscr{H}},
$$

where

$$
\sigma \mathscr{H}=\left(H_{\sigma^{-1}(1)}, \ldots, H_{\sigma^{-1(r)}}\right), \quad \sigma T=\left(t_{\sigma^{-1}(1)}, \ldots, t_{\sigma^{-1}(r)}\right)
$$

by the commutativity of the homology algebra $H_{*}\left(F\left(\mathbb{R}^{q}, \infty\right) / \mathfrak{S}_{\infty}\right)$. This leads us to define the equivalence relation $\widetilde{\mathfrak{S}}$ on $\bigsqcup_{t \geq 0} J(q)^{t} \times \mathbb{N}^{t}$ as follows. Suppose $(\mathscr{H}, T) \in J(q)^{r} \times \mathbb{N}^{r},\left(\stackrel{\mathscr{H}^{\prime}}{ }, T^{\prime}\right) \in J(q)^{s} \times \mathbb{N}^{s}$. Then $(\mathscr{H}, T) \underset{\mathfrak{S}}{\widetilde{H}}\left(\mathscr{H}^{\prime}, T^{\prime}\right)$ iff $r=s$ and there exists $\sigma \in \mathfrak{S}_{r}$ such that $\left(\mathscr{H}^{\prime}, T^{\prime}\right)=(\sigma \mathscr{H}, \sigma T)$. Each equivalence class in this relation is called an $\mathfrak{S}$-orbit.

As is easily seen, $H^{*}\left(F\left(\mathbb{R}^{q}, \infty\right) / \mathfrak{S}_{\infty}\right)$ admits the additive basis consisting of the elements

$$
W_{T}^{\mathscr{H}},(\mathscr{H}, T)=\left(H_{1}, \ldots, H_{r}\right) \times\left(t_{1}, \ldots, t_{r}\right) \in J^{+}(q)^{r} \times \mathbb{N}^{r}
$$

with $H_{1}<\cdots<H_{r}, r \geq 0$. Here $<$ denotes a certain order in $J(q)$ defined by length and by the lexicographic order for elements of the same length; where by the length of $H=\left(h_{0}, \ldots, h_{n-1}\right)$ we mean the number $l(H)=n$.

Again, the above basis is called the Dickson basis of

$$
H^{*}\left(F\left(\mathbb{R}^{q}, \infty\right) / \mathfrak{S}_{\infty}\right) .
$$

In $[13 ; 3.5]$, we defined for $(\mathscr{H}, T) \in J(\infty)^{r} \times \mathbb{N}^{r}$ and $(\mathscr{K}, U) \in$ $J(\infty)^{s} \times \mathbb{N}^{s}$ the subset $(\mathscr{H}, T) \vee(\mathscr{K}, U)$ of $\coprod_{t>0} J(\infty)^{t} \times \mathbb{N}^{t}$. We put (4.20) $(\mathscr{H}, T) \stackrel{q}{\vee}(\mathscr{K}, U)=((\mathscr{H}, T) \vee(\mathscr{K}, U)) \cap\left(\coprod_{t \geq 0} J(q)^{t} \times \mathbb{N}^{t}\right)$. 
4.21. Lemma. In terms of the Dickson basis, the structure of the Hopf algebra $H^{*}\left(F\left(\mathbb{R}^{q}, \infty\right) / \mathfrak{S}_{\infty}\right)$ is described as follows.

(i) For $(\mathscr{H}, T),(\mathscr{K}, U) \in \bigsqcup_{t \geq 0} J(q)^{t} \times \mathbb{N}^{t}$ we have

$$
W_{T}^{\mathscr{K}} \cdot W_{U}^{\mathscr{K}}=\sum_{(\mathscr{X}, Y)} W_{Y}^{\mathscr{X}}
$$

where the summation runs over the representatives of $\mathfrak{S}$-orbits of $(\mathscr{K}, T) \stackrel{q}{\vee}(\mathscr{K}, U)$.

(ii) $\Delta W_{t_{1}, \ldots, t_{r}}^{H_{1}, \ldots, H_{r}}=\sum_{u_{1}+v_{i}=t_{i}} W_{u_{1}, \ldots, u_{r}}^{H_{1}, \ldots, H_{r}} \otimes W_{v_{1}, \ldots, v_{r}}^{H_{1}, \ldots, H_{r}}$ for $H_{1}, \ldots, H_{r} \in$ $J^{+}(q)$ and $H_{1}<\cdots<H_{r}$.

The lemma can be implied from its special case where $q=\infty$ shown in $[13 ; 3.6]$ by means of Lemma 4.5 .

4.22. Definition. (i) The depth $\theta(z)$ of an element $z$ in the Dickson basis of $H^{*}\left(F\left(\mathbb{R}^{q}, \infty\right) / \mathfrak{S}_{\infty}\right)$ is defined by the formulas

$$
\theta(1)=0, \quad \theta\left(W_{t_{1}, \ldots, t_{r}}^{H_{1}, \ldots, H_{r}}\right)=\sum_{i=1}^{r} t_{i} 2^{l\left(H_{\imath}\right)}
$$

(ii) Suppose that $z=\sum_{(\mathscr{H}, T)} W_{T}^{\mathscr{H}}$ is the linear decomposition of $z \in H^{*}\left(F\left(\mathbb{R}^{q}, \infty\right) / \mathfrak{S}_{\infty}\right)$ in terms of the Dickson basis. Then we put

$$
\theta(z)=\min _{(\mathscr{H}, T)} \theta\left(W_{T}^{\mathscr{H}}\right) .
$$

Note that Lemma 4.21 enables us to compute death of arbitrary $z$.

4.23. Theorem. Let $J_{\text {odd }}(q, m)=\left\{H \in J_{\text {odd }}(q) ; 2^{l(H)} \leq m\right\}$. Then we have an isomorphism of algebras for arbitrary natural number $m$

$$
H^{*}\left(F\left(\mathbb{R}^{q}, m\right) / \mathfrak{S}_{m}\right) \cong \mathbb{Z}_{2}\left[W^{H} ; H \in J_{\text {odd }}(q, m)\right] / I(q, m)
$$

where $I(q, m)$ denotes the ideal generated by

$$
\begin{gathered}
\left\{\left(W^{H}\right)^{2^{h(q, H)}} ; H \in J_{\text {odd }}(q, m)\right\} \text { and } \\
\left\{z \in \mathbb{Z}_{2}\left[W^{H} ; H \in J_{\text {odd }}(q, m)\right] ; \theta(z)>m\right\} .
\end{gathered}
$$


The theorem can be proved easily by use of the epimorphism

$$
i^{*}(F, q): H^{*}\left(\mathfrak{S}_{m}\right)=H^{*}\left(F\left(\mathbb{R}^{\infty}, m\right) / \mathfrak{S}_{m}\right) \rightarrow H^{*}\left(F\left(\mathbb{R}^{q}, m\right) / \mathfrak{S}_{m}\right)
$$

and the result on $H^{*}\left(\mathfrak{S}_{m}\right)$ due to the author (see $[13 ; 3.8]$ ).

REMARK. From Theorems 4.15 and 4.23 we obtain the results of D. B. Fuks [5], G. Segal (see [1]) on $H^{*}\left(F\left(\mathbb{R}^{2}, m\right) / \mathfrak{S}_{m}\right)$ and of F. Cohen [2] on $H^{*}\left(F\left(\mathbb{R}^{q}, 2\right) / \mathfrak{S}_{2}\right)$.

\section{REFERENCES}

[1] E. Brieskorn, Sur les groupes de tresses, Seminaire Bourbaki, No. 401, 1971/72.

[2] F. Cohen, The Homology of $\mathscr{C}_{n+1}$-Spaces, $n \geq 0$, Springer Lecture Notes in Math., 533 (1976), 207-351.

[3] L. E. Dickson, $A$ fundamental system of invariants of the general modular linear group with a solution of the form problem, Trans. Amer. Math. Soc., 12 (1911), 75-98.

[4] E. Fadell and L. Neuwirth, Configuration spaces, Math. Scand., 10, No. 1 (1962), 111-118.

[5] D. B. Fuks, Cohomology of the braid groups with coefficients in $\mathbb{Z}_{2}$, Funktsional. Anal. i Prilozhen., 4 (1970), No. 2, 62-73.

[6] Huỳnh Mùi, Modular invariant theory and cohomology algebras of symmetric groups, J. Fac. Sci., Univ. of Tokyo, Sec. IA, 22 (1975), 319-369.

[7] _ Duality in the infinite symmetric products, Acta Math. Vietnam., 5, No. 1 (1980), 100-149.

[8] J. P. May, A General Algebraic Approach to Steenrod Operations, Springer Lecture Notes in Math., 169 (1970), 153-231.

[9] _ The Geometry of Iterated Loop Spaces, Springer Lecture Notes in Math., 271 (1972).

[10] _ The Homology of $E_{\infty}$ Spaces, Springer Lecture Notes in Math., 533 (1976), 1-68.

[11] T. Nakamura, On cohomology operation, Japan. J. Math., 33 (1963), 93-145.

[12] M. Nakaoka, Homology of the infinite symmetric group, Ann. of Math., 73 (1961), 229-257.

[13] Nguy ên Hũu Việt Hung, The mod 2 cohomology algebras of symmetric groups, Acta Math. Vietnam., 6, No. 2 (1981), 41-48.

[14] _ The mod 2 equivariant cohomology algebras of configuration spaces, Acta Math. Vietnam., 7, No. 1 (1982), 95-100.

[15] _ The modulo 2 cohomology algebras of symmetric groups, Japan. J. Math., 13, No. 1 (1987), 169-208.

[16] __, Algèbre de cohomologie du groupe symétrique infini et classes caractéristiques de Dickson, C. R. Acad. Sci. Paris, t. 297, Série I (1983), 611-614.

[16b] Nguyên H. V. Hung, Classes de Dickson et algèbres de cohomologie de espaces de lacets itérés, C. R. Acad. Sci. Paris Série I, 307 (1988), 911-914.

[16c] Nguyên H. V. Hung and Nguyên N. Hai, Steenrod operations on mod 2 homology of the iterated loop space $\Omega^{q} S^{q}$, Acta Math. Vietnam., to appear.

[17] D. Quillen, The Adams conjecture, Topology, 10 (1971), 67-80.

[18] _ The mod 2 cohomology rings of extra-special 2-groups and the spinor groups, Math. Ann., 11 (1972), 197-212. 
[19] G. Segal, Configuration spaces and iterated loop spaces, Invent. Math., 21 (1973), 213-221.

[20] N. E. Steenrod and D. B. A. Epstein, Cohomology operations, Ann. of Math. Studies No. 50, Princeton Univ. Press (1962).

[21] R. Wellington, The unstable Adams spectral sequence for free iterated loop spaces, Memoirs of the Amer. Math. Soc., 36 (1982).

Received January 8, 1987 and in revised form March 1, $1988^{1}$.

UNIVERSITY OF HANOI

DAI HOC TONG hOP, HANOI, VIETNAM

${ }^{1}$ According to a letter from the author dated 2/7/90, this paper was finished in August 1982 and given by Professor H. Mui, the author's advisor, to Professor H. Miller in the Spring of 1985 during the Topology Conference at the University of Washington.-The Editor 


\section{PACIFIC JOURNAL OF MATHEMATICS EDITORS}

\author{
V. S. VARADARAJAN \\ (Managing Editor) \\ University of California \\ Los Angeles, CA 90024-1555-05 \\ Herbert Clemens \\ University of Utah \\ Salt Lake City, UT 84112 \\ ThOMAs ENRIGHT \\ University of California, San Diego \\ La Jolla, CA 92093
}

R. FINN

Stanford University

Stanford, CA 94305

HeRmanN FlaschKa

University of Arizona

Tucson, AZ 85721

VAUGHAN F. R. JoNES

University of California

Berkeley, CA 94720

SteVen KerCKhofF

Stanford University

Stanford, CA 94305
ROBION KIRBY

University of California

Berkeley, CA 94720

C. C. Moore

University of California

Berkeley, CA 94720

HAROLD STARK

University of California, San Diego

La Jolla, CA 92093

\section{ASSOCIATE EDITORS}
R. ARENS
E. F. BECKENBACH
B. H. NeumanN
F. Wolf
K. YoshidA
(1906-1982)
(1904-1989)

\section{SUPPORTING INSTITUTIONS}

UNIVERSITY OF ARIZONA
UNIVERSITY OF BRITISH COLUMBIA
CALIFORNIA INSTITUTE OF TECHNOLOGY
UNIVERSITY OF CALIFORNIA
MONTANA STATE UNIVERSITY
UNIVERSITY OF NEVADA, RENO
NEW MEXICO STATE UNIVERSITY
OREGON STATE UNIVERSITY
UNIVERSITY OF ARIZONA
UNIVERSITY OF BRITISH COLUMBIA
UNIVERSITY OF CALIFORNIA
MONTANA STATE UNIVERSITY
NEW MEXICO STATE UNIVERSITY

\author{
UNIVERSITY OF OREGON \\ UNIVERSITY OF SOUTHERN CALIFORNIA \\ STANFORD UNIVERSITY \\ UNIVERSITY OF HAWAII \\ UNIVERSITY OF TOKYO \\ UNIVERSITY OF UTAH \\ WASHINGTON STATE UNIVERSITY \\ UNIVERSITY OF WASHINGTON
}

The Supporting Institutions listed above contribute to the cost of publication of this Journal, but they are not owners or publishers and have no responsibility for its content or policies.

Mathematical papers intended for publication in the Pacific Journal of Mathematics should be in typed form or offset-reproduced (not dittoed), double spaced with large margins. Please do not use built up fractions in the text of the manuscript. However, you may use them in the displayed equations. Underline Greek letters in red, German in green, and script in blue. The first paragraph must be capable of being used separately as a synopsis of the entire paper. In particular it should contain no bibliographic references. Please propose a heading for the odd numbered pages of less than 35 characters. Manuscripts, in triplicate, may be sent to any one of the editors. Please classify according to the 1980 Mathematics Subject Classification (1985 Revision) scheme which can be found in the December index volumes of Mathematical Reviews. Supply name and address of author to whom proofs should be sent. All other communications should be addressed to the managing editor, or Elaine Barth, University of California, Los Angeles, California 90024-1555-05.

There are page-charges associated with articles appearing in the Pacific Journal of Mathematics. These charges are expected to be paid by the author's University, Government Agency or Company. If the author or authors do not have access to such Institutional support these charges are waived. Single authors will receive 50 free reprints; joint authors will receive a total of 100 free reprints. Additional copies may be obtained at cost in multiples of 50 .

The Pacific Journal of Mathematics is issued monthly as of January 1966. Regular subscription rate: $\$ 190.00$ a year (6 Vols., 12 issues). Special rate: $\$ 95.00$ a year to individual members of supporting institutions.

Subscriptions, orders for numbers issued in the last three calendar years, and changes of address should be sent to Pacific Journal of Mathematics, P.O. Box 969, Carmel Valley, CA 93924, U.S.A. Old back numbers obtainable from Kraus Periodicals Co., Route 100, Millwood, NY 10546.

The Pacific Journal of Mathematics at P.O. Box 969, Carmel Valley, CA 93924 (ISSN 0030-8730) is published monthly. Second-class postage paid at Carmel Valley, California 93924, and additional mailing offices. Postmaster: send address changes to Pacific Journal of Mathematics, P.O. Box 969, Carmel Valley, CA 93924.

\section{PUBLISHED BY PACIFIC JOURNAL OF MATHEMATICS, A NON-PROFIT CORPORATION}




\section{Pacific Journal of Mathematics}

\section{Vol. 143, No. $2 \quad$ April, 1990}

Gustavo Corach, Horacio Porta and Lázaro Recht, Differential geometry of systems of projections in Banach algebras ................. 209

Peter Fleischmann and Jens Carsten Jantzen, Simple periodic modules of

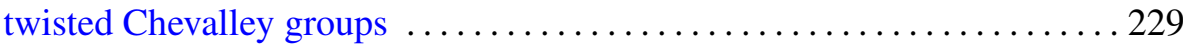

Niels Gronbaek, Amenability of discrete convolution algebras, the commutative case ...................................243

Nguyên H. V. Hung, The mod 2 equivariant cohomology algebras of

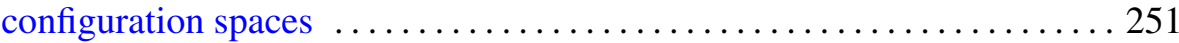

Wojciech Kucharz, Global almost analytic algebraicity of analytic sets . . . 287 John Merrill, A class of consistent anti-Martin's axioms .............. 301

Takafumi Murai, The power 3/2 appearing in the estimate of analytic

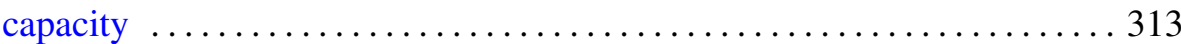

L. Panaitopol and Doru Stefanescu, On the generalized difference

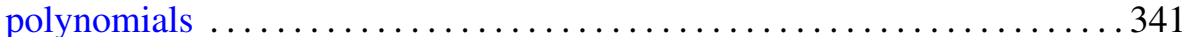

Katsuhiro Shiohama, Takashi Shioya and Minoru Tanaka, Mass of rays

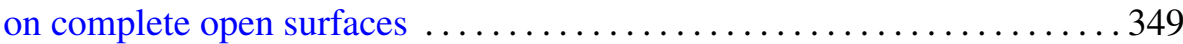

Gerhard Ströhmer, About compressible viscous fluid flow in a bounded

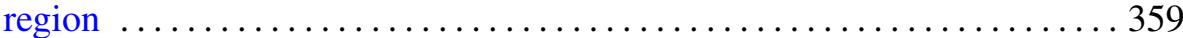

A. Ülger, Arens regularity sometimes implies the RNP 